\title{
Intestinal farnesoid $X$ receptor signaling promotes nonalcoholic fatty liver disease
}

\author{
Changtao Jiang, ${ }^{1,2}$ Cen Xie, ${ }^{1}$ Fei Li, ${ }^{1}$ Limin Zhang, ${ }^{3,4}$ Robert C. Nichols, ${ }^{3}$ Kristopher W. Krausz, ${ }^{1}$ Jingwei Cai, ${ }^{3}$ Yunpeng Qi, \\ Zhong-Ze Fang, ${ }^{1}$ Shogo Takahashi, ${ }^{1}$ Naoki Tanaka, ${ }^{1}$ Dhimant Desai, ${ }^{5}$ Shantu G. Amin, ${ }^{5}$ Istvan Albert, ${ }^{6}$ \\ Andrew D. Patterson, ${ }^{3}$ and Frank J. Gonzalez ${ }^{1}$ \\ 'Laboratory of Metabolism, Center for Cancer Research, National Cancer Institute (NCI), NIH, Bethesda, Maryland, USA. Department of Physiology and Pathophysiology, School of Basic Medical Sciences, \\ Peking University, Key Laboratory of Molecular Cardiovascular Science, Ministry of Education, Beijing, China. ${ }^{3}$ Department of Veterinary and Biomedical Sciences and the Center for Molecular Toxicology \\ and Carcinogenesis, The Pennsylvania State University, University Park, Pennsylvania, USA. " CAS Key Laboratory of Magnetic Resonance in Biological Systems, State Key Laboratory of Magnetic Resonance, \\ Wuhan Institute of Physics and Mathematics, Chinese Academy of Sciences, Wuhan, China. ${ }^{5}$ Department of Pharmacology, College of Medicine, The Pennsylvania State University, Hershey, \\ Pennsylvania, USA. ${ }^{6}$ Bioinformatics Consulting Center, The Pennsylvania State University, University Park, Pennsylvania, USA.
}

\begin{abstract}
Nonalcoholic fatty liver disease (NAFLD) is a major worldwide health problem. Recent studies suggest that the gut microbiota influences NAFLD pathogenesis. Here, a murine model of high-fat diet-induced (HFD-induced) NAFLD was used, and the effects of alterations in the gut microbiota on NAFLD were determined. Mice treated with antibiotics or tempol exhibited altered bile acid composition, with a notable increase in conjugated bile acid metabolites that inhibited intestinal farnesoid $X$ receptor (FXR) signaling. Compared with control mice, animals with intestine-specific Fxr disruption had reduced hepatic triglyceride accumulation in response to a HFD. The decrease in hepatic triglyceride accumulation was mainly due to fewer circulating ceramides, which was in part the result of lower expression of ceramide synthesis genes. The reduction of ceramide levels in the ileum and serum in tempol- or antibiotic-treated mice fed a HFD resulted in downregulation of hepatic SREBP1C and decreased de novo lipogenesis. Administration of C16:0 ceramide to antibiotic-treated mice fed a HFD reversed hepatic steatosis. These studies demonstrate that inhibition of an intestinal FXR/ceramide axis mediates gut microbiota-associated NAFLD development, linking the microbiome, nuclear receptor signaling, and NAFLD. This work suggests that inhibition of intestinal FXR is a potential therapeutic target for NAFLD treatment.
\end{abstract}

\section{Introduction}

Nonalcoholic fatty liver disease (NAFLD) is characterized by massive ectopic triglyceride accumulation in the liver in the absence of other liver disease or significant alcohol consumption (1). NAFLD is the most common liver disorder, affecting $20 \%-30 \%$ of the adult population and more than $80 \%$ of obese people in the world. NAFLD can develop into nonalcoholic steatohepatitis (NASH), fibrosis, cirrhosis, and hepatocellular carcinoma (2). As a component of metabolic syndrome, NAFLD is tightly associated with insulin resistance, type 2 diabetes, coronary heart disease, and atherosclerosis (3). In addition, NAFLD is a risk factor for hepatocellular carcinoma (4). To date, the underlying molecular mechanism of NAFLD pathogenesis remains largely unknown, and the identification of novel targets for NAFLD therapy is of high priority.

Recent studies have implicated the gut microbiota in the development of NAFLD in mice and humans (5). Oral treatment of lean germ-free mice with the cecal microbiota of obese mice caused an increase in hepatic triglyceride accumulation (6). Further, obese humans are enriched in the microbial energy-harvesting phylum Firmicutes, which can directly improve energy yield from intestinal contents to accelerate obesity associated with NAFLD (7). Other

Conflict of interest: The authors have declared that no conflict of interest exists Submitted: April 28, 2014; Accepted: November 6, 2014

Reference information: / Clin Invest. 2015;125(1):386-402. doi:10.1172/JCI76738. studies have revealed an association between the gut microbiota and metabolism in NAFLD $(8,9)$. Possible mechanisms by which the gut microbiota and their metabolites could influence NAFLD have been reviewed (10). More recent studies in mice reported that alteration of the gut microbiota changes host bile acid composition, notably alteration of taurine-conjugated bile acids that can antagonize the intestinal farnesoid X receptor (FXR) $(11,12)$ and could give rise to metabolic dysfunction including obesity and insulin resistance (13). Bile acids could also influence NAFLD through activation of the hepatic FXR and the G protein-coupled receptor (GPCR) TGR5 expressed in nonparenchymal cells (14). However, questions remain about the roles of the gut microbiota, bile acids, and intestinal and hepatic FXR signaling in the pathogenesis of hepatic steatosis.

In the current study, mice were fed a high-fat diet (HFD) to induce NAFLD. A combination of bacitracin, neomycin, and streptomycin (BNS) to kill certain members of the gut microbiota, or tempol treatment to specifically modulate the gut microbiota, was used to determine the role of the gut microbiota in NAFLD pathogenesis. Intestine-specific Fxr-null (villin-Cre Fxr fl/fl, referred to hereafter as $F x r^{I I E}$ ) mice were used to elucidate the mechanism by which the gut microbiota contributes to NAFLD. This study demonstrated that the gut microbiota influences NAFLD pathogenesis by regulating the bile acid/intestinal FXR axis that alters the ceramide/SREBP1C/CIDEA pathway and, combined with 
A
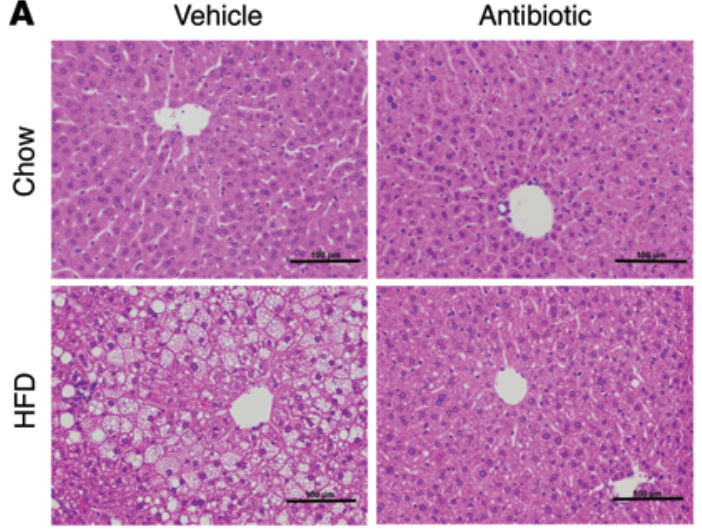

C

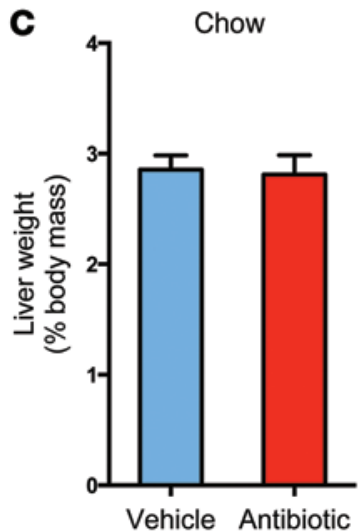

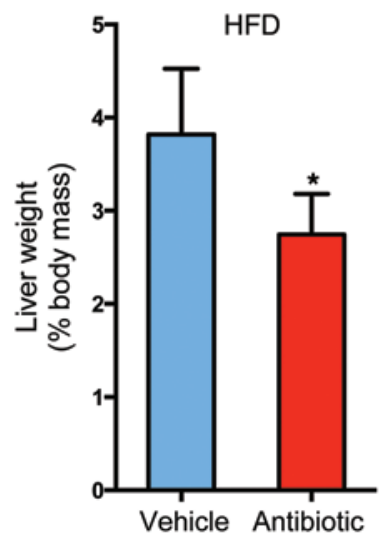

B

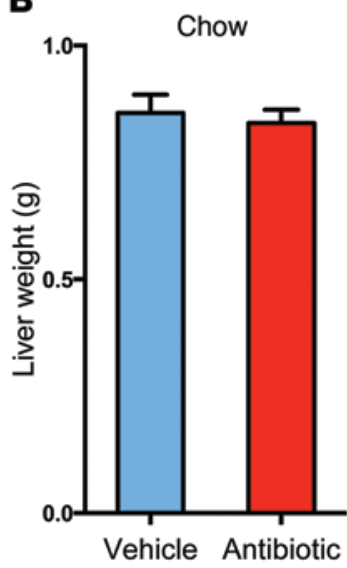

HFD

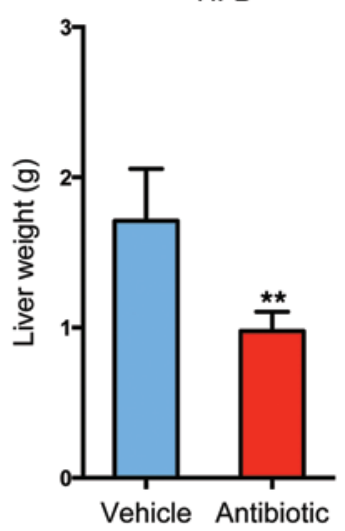

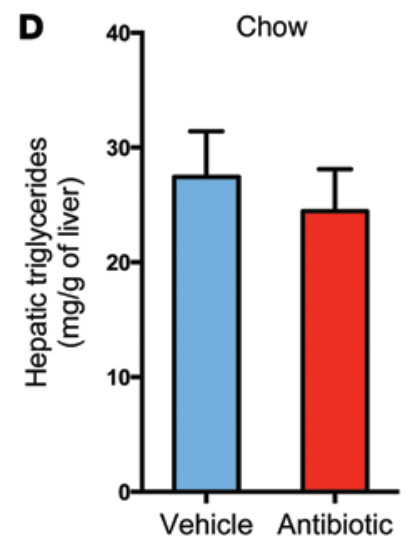

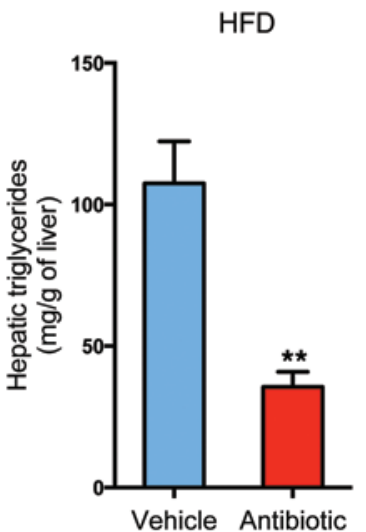

Figure 1. Antibiotic treatment ameliorates the development of NAFLD. (A) Representative H\&E staining of liver sections from vehicle- and antibiotic-treated mice on a chow diet or a HFD for 7 weeks. Scale bars: $100 \mu \mathrm{m} . n=5$ mice per group. (B) Liver weights of vehicle- and antibiotic-treated mice on a chow diet or a HFD for 7 weeks. $n=5$ mice per group. (C) Ratios of liver weight/body weight of vehicle- and antibiotic-treated mice on a chow diet or a HFD for 7 weeks. $n=5$ mice per group. (D) Liver triglyceride content of vehicle- and antibiotic-treated mice on a chow diet or a HFD for 7 weeks. $n=5$ mice per group. All data are presented as the mean \pm SD. ${ }^{*} P<0.05$ and ${ }^{* *} P<0.01$ (2-tailed Student's $t$ test) compared with vehicle-treated mice.

reduced bacterial fermentation in the gut, results in decreased hepatic lipids. Furthermore, this work reveals an essential role for intestinal FXR in controlling liver lipid metabolism and provides a potential therapeutic strategy for the prevention of disease progression in patients with NAFLD.

\section{Results}

A HFD is extensively used as a mouse model for NAFLD. The antioxidant tempol selectively modulates the gut microbiota composition and metabolism under normal and HFD conditions (12). In an effort to determine whether tempol modifies the gut microbiota in the HFD-induced NAFLD model, 16S rRNA gene-sequencing analysis was performed. Weighted UniFrac analysis showed distinct clustering of cecal communities isolated from vehicle- and tempol-treated groups on a HFD for 16 weeks, demonstrating a significant change in bacterial abundance (Supplemental Figure $1 \mathrm{~A}$; supplemental material available online with this article; doi:10.1172/JCI76738DS1). The separation of samples in the principal components analysis (PCA) plot likely reflects decreased Firmicutes and markedly increased Proteobacteria (Supplemental Figure 1B). Tempol treatment had no effect on total bacterial counts as measured by quantitative PCR (qPCR) (Supplemental
Figure 1C). The genus Desulfovibrio was identified as a major contributor of the increased Proteobacteria (Supplemental Figure 1D), which were found in substantially lower numbers in obese human subjects (15). A dramatic increase was observed in the genus Roseburia (Supplemental Figure 1E) that was negatively correlated with body weight (16). In addition, the levels of the genus Clostridium sensu stricto and Lactobacillus were also substantially decreased in tempol-treated mice, whereas the levels of the genus Bacteroides and Streptococcus remained similar (Supplemental Figure 1, F-I).

Following the change in gut microbiota composition, liver histology indicated a marked reduction in hepatic lipid droplets in tempol-treated mice on a HFD for 16 weeks and antibiotic-treated mice on a HFD for 7 weeks (Supplemental Figure 2, A and B, and Figure 1A). Tempol treatment or antibiotic treatment decreased liver weights and liver/body mass ratios in mice fed a HFD (Supplemental Figure 2, C and D, and Figure 1, B and C). Hepatic triglyceride contents were decreased to approximately $50 \%$ and $35 \%$ in mice treated with tempol or antibiotics on a HFD, respectively (Supplemental Figure 2E and Figure 1D). Tempol or antibiotic treatment had no obvious effects on liver morphology, liver weights, liver weight/body weight ratios, or hepatic triglyceride content in mice on a normal chow diet (Supplemental Figure 2, A 


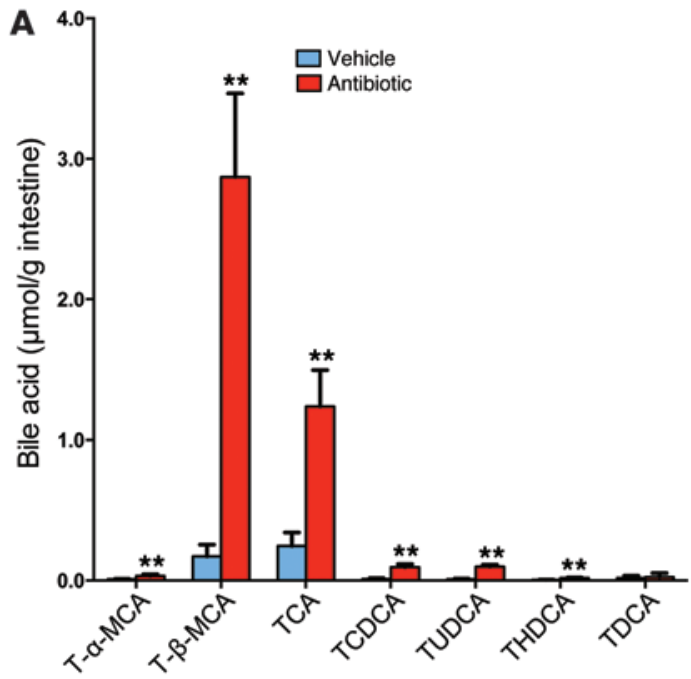

B
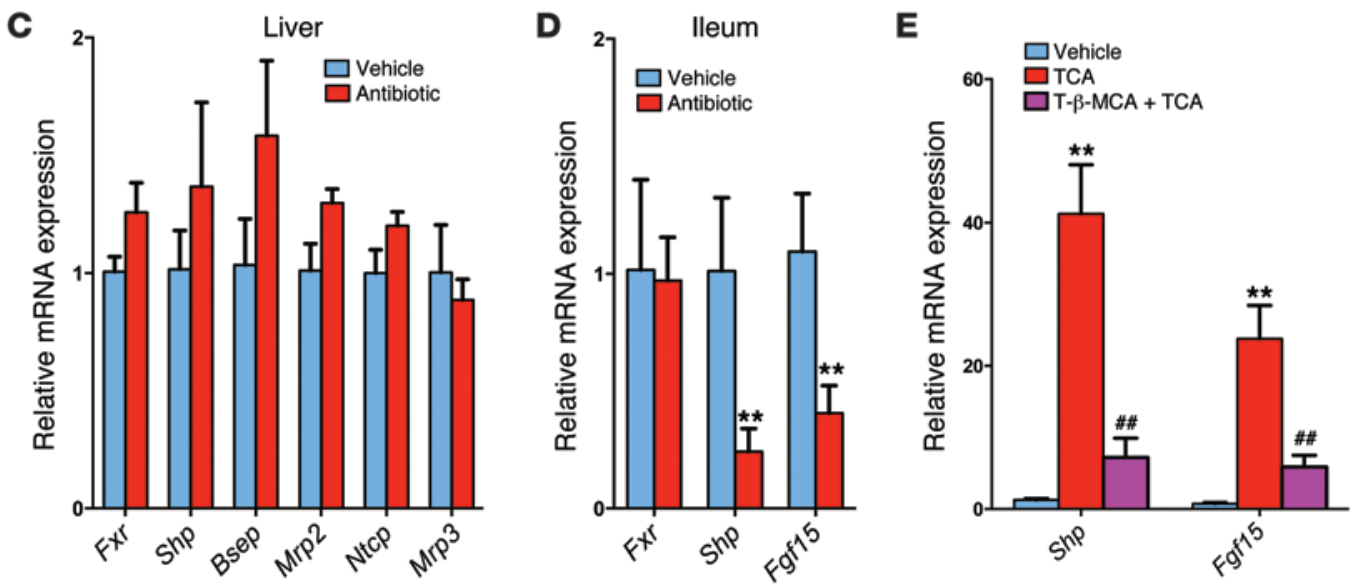

Figure 2. Gut microbiota regulate bile acid composition. (A) Individual taurine-conjugated bile acid levels in the ileum of HFD-fed mice after 7 weeks of antibiotic treatment. $n=4-5$ mice per group. (B) Proportion of individual taurine-conjugated bile acids, expressed as a percentage of the total bile acid pool, in ileum from the vehicle- and antibiotic-treated groups fed a HFD for 7 weeks. $n=4-5$ mice per group. (C) mRNA levels for FXR signaling-related genes in the liver after 7 weeks of antibiotic treatment in mice fed a HFD. $n=4-5$ mice per group. (D) mRNA levels of Shp and Fgf15 in ileum after 7 weeks of antibiotic treatment in HFD-fed mice. $n=5$ mice per group. (C and D) Expression was normalized to 18S RNA. (A-D) Data are presented as the mean \pm SD. ${ }^{*} P<0.01$ (2-tailed Student's $t$ test) compared with vehicle-treated mice. (E) mRNA levels of Shp and Fgf15 in ileum after 24 hours of T- $\beta$-MCA with TCA treatment in HFD-fed mice. mRNA expression was normalized to 18S RNA. $n=6$ mice per group. Data are presented as the mean \pm SD. One-way ANOVA with Tukey's correction. ${ }^{* *} P<0.01$ compared with vehicle; ${ }^{\# \#} P<0.01$ compared with TCA treatment.

and C-E, and Figure 1, A-D). To validate the role of the gut microbiota in tempol-reduced NAFLD, vehicle and antibiotic-treated mice fed a HFD were also subjected to tempol treatment for 16 weeks. Liver histology revealed that hepatic lipid droplets in tempol-treated, antibiotic-treated, and tempol plus antibiotictreated mice were substantially reduced compared with the droplets observed in vehicle-treated mice fed a HFD for 16 weeks (Supplemental Figure 3A). No significant decreases in liver weights, liver/body mass ratios, or hepatic triglyceride content were observed when tempol was combined with antibiotics beyond what was found with antibiotic treatment alone (Supplemental Figure 3, B and C). These results suggested that changes in the gut microbiota were correlated with NAFLD.

Gut microbiota modifies bile acid metabolism and FXR signaling. The gut microbiota is tightly associated with bile acid metabolism. UPLC-ESI-QTOFMS (ultra-performance liquid chromatography- electrospray ionization-quadrupole time-of-flight mass spectrometry) metabolomics analysis was adopted to determine bile acid composition and levels of bile acid metabolites in the intestine. A scores scatter plot of a PCA model of the UPLC-ESI-QTOFMS negative mode data from mouse ileum indicated distinct metabolic profiles between the vehicle- and antibiotic-treated groups (Supplemental Figure 4A). The top enriched metabolite tauro$\beta$-muricholic acid (T- $\beta$-MCA, $m / z 514.2871$, retention time $=6.64$ minutes) was increased in the antibiotic-treated mice on a HFD for 7 weeks, as revealed in the loading scatter plot (Supplemental Figure 4B) established according to previously described methods (12). Liquid chromatography-tandem mass spectrometric (LC-MS/ MS) quantitation confirmed that the levels of taurine-conjugated bile acids were increased after antibiotic treatment, and the levels of T- $\beta$-MCA were increased 17-fold in antibiotic-treated mice on a HFD for 7 weeks (Figure 2A). Other taurine-conjugated bile 
A
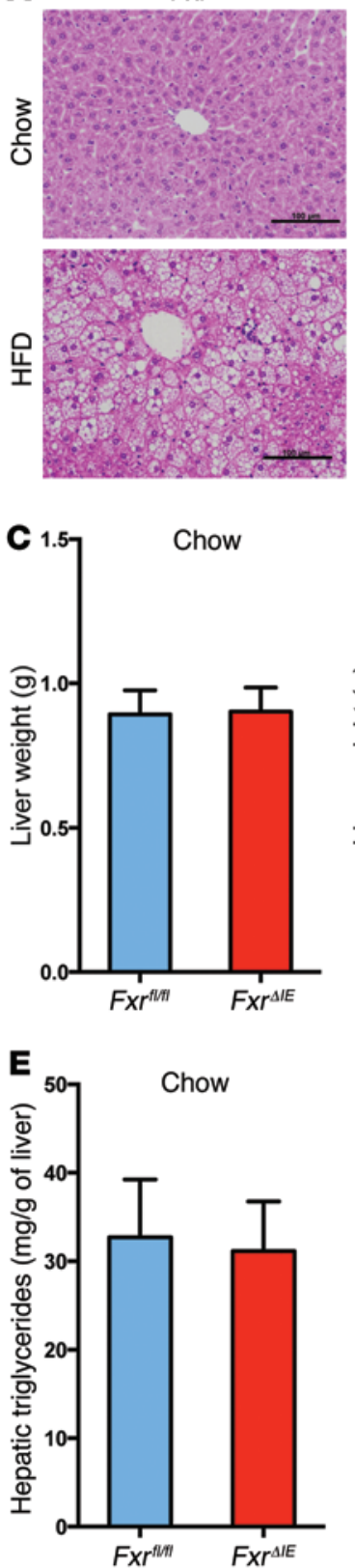

Fxr ${ }^{\Lambda E}$
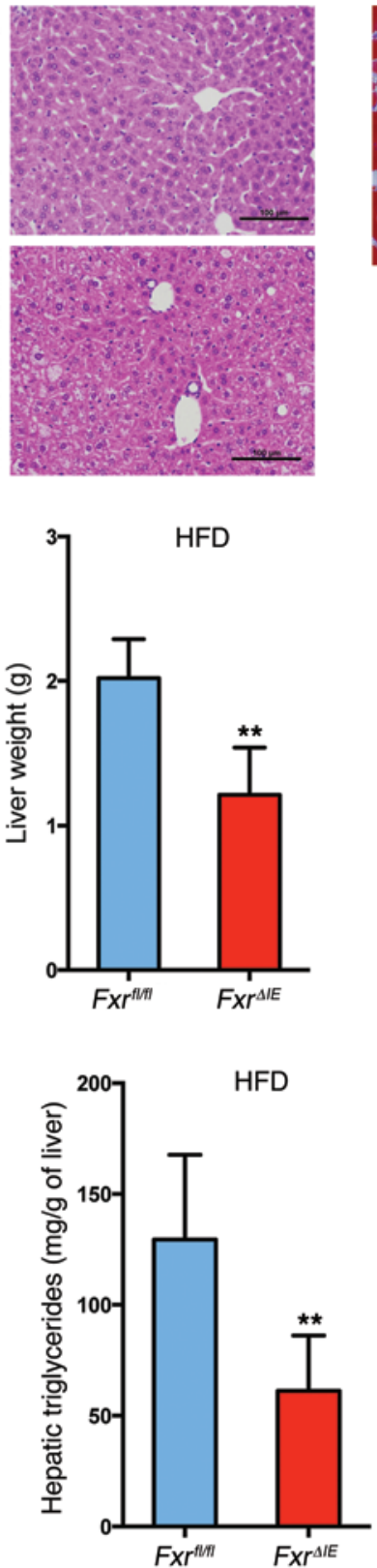

B

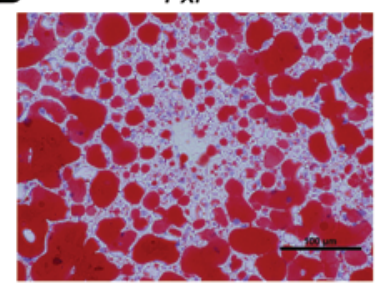

Fxr ${ }^{m ! n}$

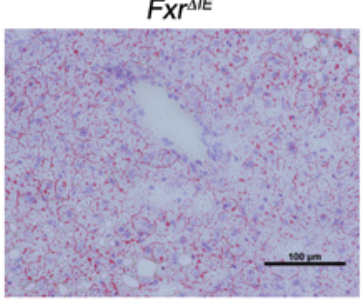

D
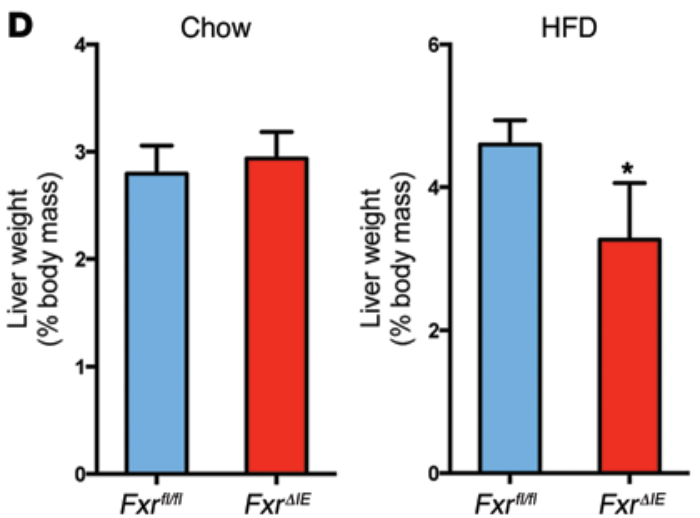

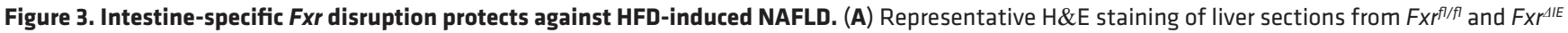
mice after 14 weeks of chow or HFD feeding. Scale bars: $100 \mu \mathrm{m} . n=4-5$ mice per group. (B) Representative Oil red 0 staining of liver sections from Fxr ${ }^{7 / f l}$ and FXr ${ }^{\Delta I E}$ mice after 14 weeks of HFD feeding. Lipids stained positive (red color) with Oil Red O. Scale bars: $100 \mu$ m. $n=5$ mice per group. (C) Liver weights of $F x^{f / f l}$ and $F x r^{1 / E}$ mice after 14 weeks of chow or HFD feeding. $n=4-5$ mice per group. (D) Liver weight/body weight ratios of $F x^{f / / f l}$ and $F x^{\Delta / E}$ mice after 14 weeks of chow or HFD feeding. $n=4-5$ mice per group. (E) Liver triglyceride contents in FXr ${ }^{f / f l}$ and $F x r^{4 / E}$ mice after 14 weeks of chow or HFD feeding. $n=4-5$ mice per group. All data are presented as the mean \pm SD. ${ }^{*} P<0.05$ and ${ }^{* *} P<0.01$ (2-tailed Student's $t$ test) compared with $F \times r^{f / / f l}$ mice.

acids were also increased. The ratio of T- $\beta$-MCA to total bile acids was also markedly increased in antibiotic-treated mice on a HFD (Figure 2B). Similar results were obtained from tempol-treated mice on a HFD for 16 weeks (Supplemental Figure 5, A and B). The gut microbiota can modify bile acid composition by microbial enzymatic activities (17). Bile salt hydrolase (BSH) is a bacterial enzyme that hydrolyzes taurine-conjugated bile acids into free bile acids. BSH activity was almost absent in antibiotic-treated mice fed a HFD for 7 weeks (Supplemental Figure 5C). The most enriched bile acid in the ileum of antibiotic- and tempol-treated mice on a HFD was T- $\beta$-MCA, an FXR antagonist $(11,12)$. Western blot and qPCR analysis indicated that FXR protein levels were induced after 12 weeks of HFD feeding and that FXR signaling in the ileum was increased, as revealed by an increase in the FXR target gene mRNAs small heterodimer partner $(S h p)$ and fibroblast growth factor 15 (Fgf15) (Supplemental Figure 5, D and E). Conversely, antibiotic treatment inhibited FXR signaling in the ileum, but not in the liver (Figure 2, C and D). The question arose as to 
A

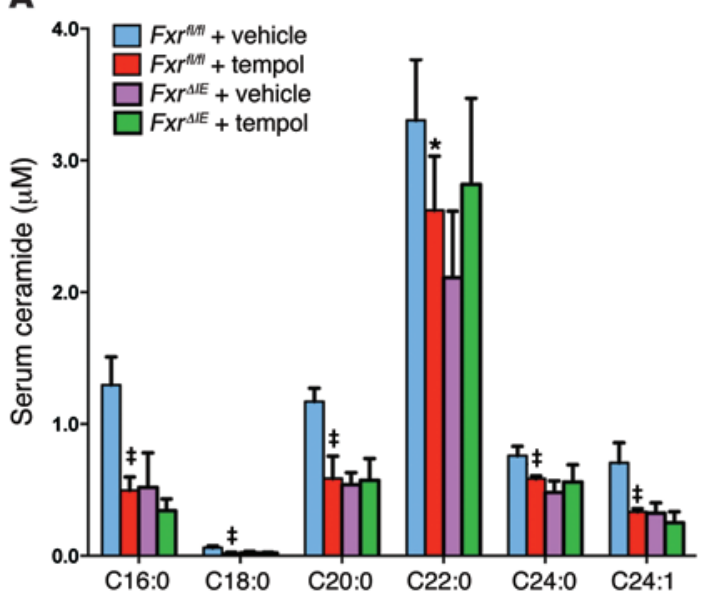

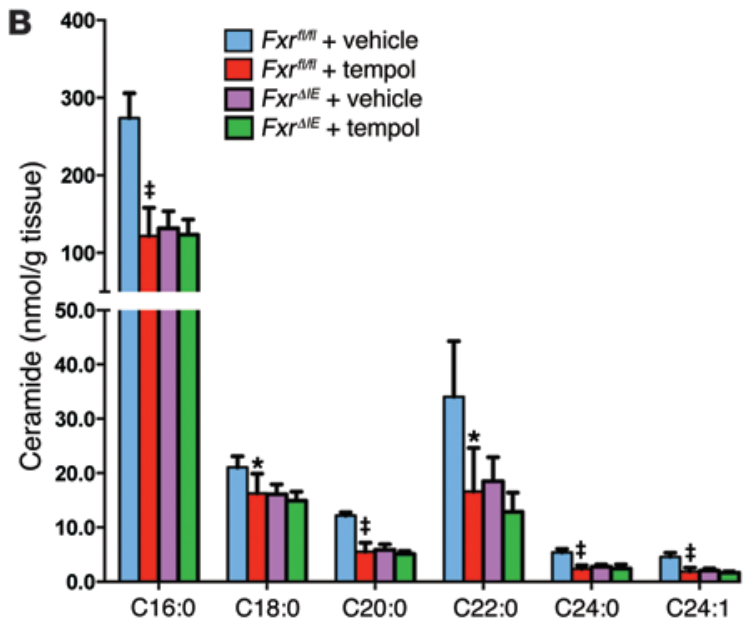
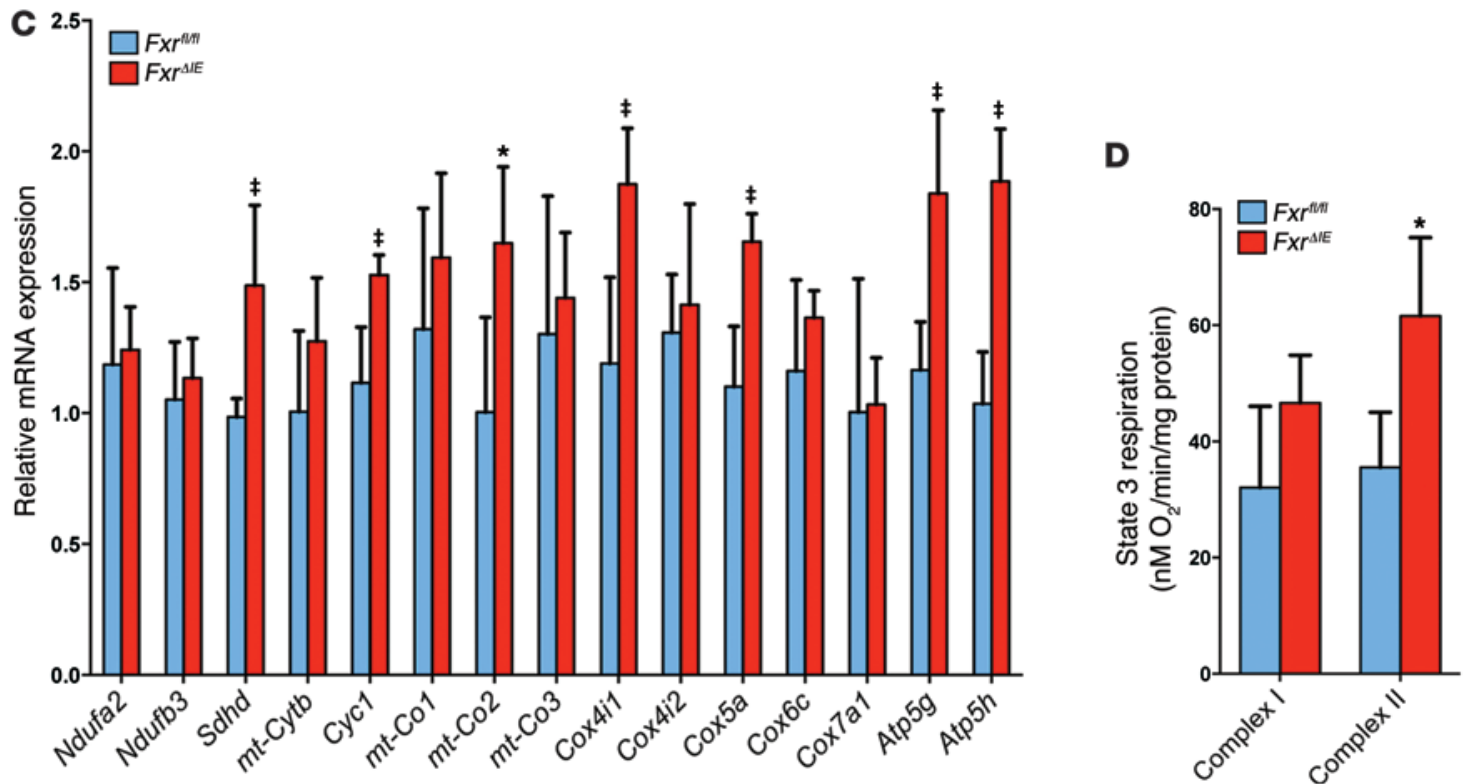

Figure 4. Intestine-specific Fxr disruption promotes mitochondrial biogenesis in the intestine. (A) Serum ceramide levels in vehicle- and tempol-treated

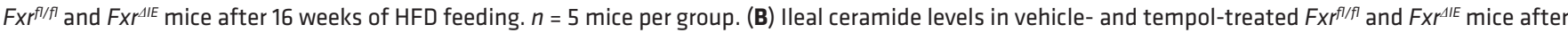
16 weeks of HFD feeding. $n=5$ mice per group. (A and B) Data are presented as the mean \pm SD. One-way ANOVA with Tukey's correction. ${ }^{*} P<0.05$ and ${ }^{\ddagger} P<0.01$ compared with vehicle-treated mice of the same genotype. C, side chain carbon number. (C) mRNA levels of mitochondrial oxidative phosphorylation-related enzymes in ileal mucosa from $F x r^{f / f f l}$ and $F x x^{1 / E}$ mice after 14 weeks of HFD feeding. Expression was normalized to $18 \mathrm{~S}$ RNA. $n=5$ mice per group. $\mathrm{mt}$, mitochondrial. (D) Measurement of state 3 respiration for complex I- and complex II-dependent respiration from the ileal mucosa of $F x r^{f / f l}$ and FXr ${ }^{\Delta I E}$ mice after 14 weeks of HFD feeding. $n=4$ mice per group. (C and D) Data are presented as the mean \pm SD. ${ }^{*} P<0.05$ and ${ }^{\ddagger} P<0.01$ (2-tailed Student's $t$ test) compared with $F x r^{f / f l}$ mice.

whether T- $\beta$-MCA inhibited FXR signaling in vivo in mice on a HFD treatment. Indeed, T- $\beta$-MCA treatment significantly blunted Shp and Fgf15 mRNA induction by taurocholic acid (TCA) in the ileum of antibiotic-treated mice on a HFD for 3 days (Figure 2E). These results indicated that antibiotic or tempol treatments regulate bile acid composition mainly by increasing T- $\beta$-MCA as a result of lower bacterial BSH activity.

Intestine-specific Fxr disruption reduces hepatic lipid accumulation. To further clarify the role of intestinal FXR in the development of NAFLD, intestine-specific Fxr-null $\left(F x r^{\triangle I E}\right)$ mice were treated with a chow diet or a HFD for 14 weeks. Fxr ${ }^{I I E}$ mice displayed no obvious phenotype when compared with WT $\left(F x r^{A / f f}\right)$ mice on a chow diet (Figure 3, A and C-E). However, upon HFD feeding, H\&E and Oil red O staining of liver sections revealed a significant decrease in hepatic lipid droplets in Fxr ${ }^{A I E}$ mice com-

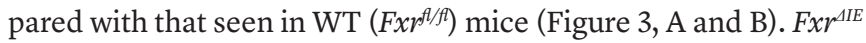
mice displayed marked reductions in both liver weights and liver weight/body weight ratios when fed a HFD (Figure 3, C and D). Hepatic triglyceride levels were 50\% lower in Fxp ${ }^{1 I E}$ mice compared with those in $F x x^{f / f}$ mice on a HFD for 14 weeks (Figure 3E). In contrast, mice lacking expression of FXR in the liver (Alb-Cre $F x^{f / f /}$, also referred to as Fxp ${ }^{\text {liv }}$ mice) showed no difference in liver weights, liver weight/body weight ratios, or hepatic triglycerides as compared with those of WT mice, even though they had lower FXR signaling as revealed by mRNA levels from FXR target genes (C. Jiang, C. Xie, S. Takahashi, and F.J. Gonzalez, unpublished 

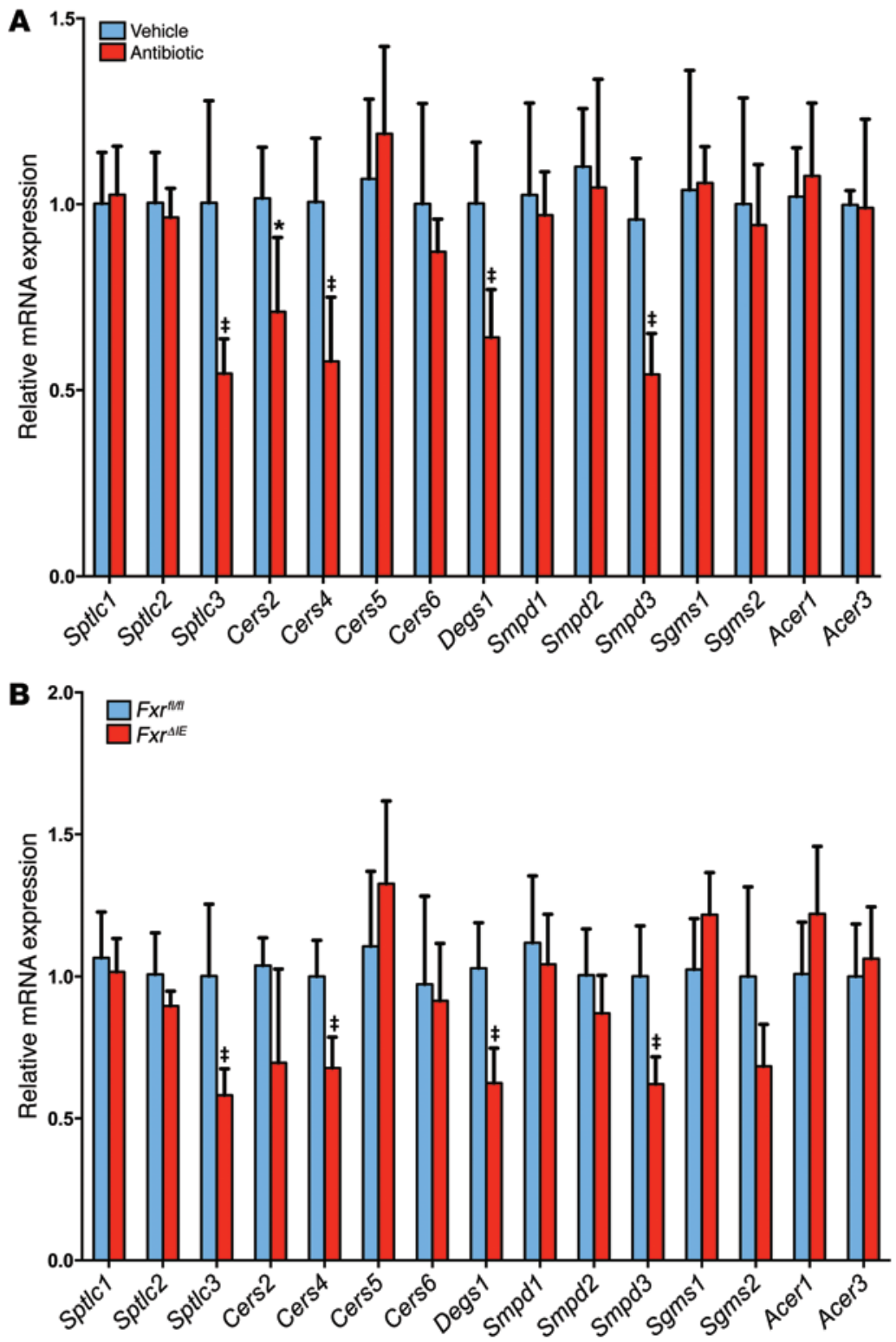

Figure 5. Inhibition of intestinal FXR alters expression of mRNAs encoding ceramide synthesis- and catabolismrelated enzymes. (A) mRNA levels for ceramide synthesisand catabolism-related enzymes in the ileum after 7 weeks of antibiotic treatment in HFD-fed mice.

$n=5$ mice per group. Data are presented as the mean \pm SD. ${ }^{*} P<0.05$ and ${ }^{\ddagger} P<0.01$ (2-tailed Student's $t$ test) compared with vehicle-treated mice. (B) mRNA levels for ceramide synthesis- and catabolism-related enzymes in the ileum of $F x r^{f / f l}$ and $F x r^{\Delta / E}$ mice fed a HFD for 14 weeks. $n=5$ mice per group. Expression was normalized to 185 RNA. Data are presented as the mean $\pm \mathrm{SD} .{ }^{*} P<0.05$ and ${ }^{\ddagger} P<0.01$ (2-tailed Student's $t$ test) compared with $F \mathrm{Xr}^{\mathrm{fl} / \mathrm{fl}}$ mice.

reduced in antibiotic-treated WT mice fed a HFD for 7 weeks (Supplemental Figure 7, B and C).

Since mitochondria contain enzymes responsible for the synthesis and hydrolysis of ceramides (19), the expression of mRNAs encoded by genes involved in mitochondrial function in the ileum of $\mathrm{Fxr}^{A / A}$ and $\mathrm{Fxr}^{A I E}$ mice was measured. The messenger RNAs encoding succinate dehydrogenase complex; subunit D; integral membrane protein $(S d h d)$; the complex III-related gene cytochrome $\mathrm{c} 1(\mathrm{Cyc1})$; the complex IV-related gene mitochondrially encoded cytochrome c oxidase II ( $m t-\mathrm{Co} 2)$; cytochrome c oxidase subunit IV isoform 1 (Cox4i1); cytochrome c oxidase subunit Va $(\operatorname{Cox} 5 a)$; ATP synthase, $\mathrm{H}^{+}$ transporting, mitochondrial FO complex, subunit $\mathrm{C} 1$ (subunit 9) (Atp5g); ATP synthase, $\mathrm{H}^{+}$transporting, mitochondrial F0 complex, and subunit D (Atp5h) were elevated in the ileal epithelium of Fxr ${ }^{I I}$ mice (Figure $4 \mathrm{C}$ ). Similar changes in gene expression were found in the ileum of antibiotic-treated mice (Supplemental Figure 7D). An approximately 70\% increased activity of complex II and no significant elevation in activity of complex I were observed in the ileal mitochondria of Fxr ${ }^{\Delta I E}$ mice compared with that seen in $\mathrm{Fxr}^{\mathrm{A} / \mathrm{f}}$ mice (Figure 4D). Ileal ATP lev-

observations). No significant alterations were found in food consumption, intestinal lipid content, or fecal lipid levels in vehicleand tempol-treated $F x r^{A / f}$ and $F x r^{A I E}$ mice fed a HFD for 16 weeks (Supplemental Figure 6, A-C). These results suggest that intestine-specific FXR inhibition had negligible effects on lipid absorption from the HFD.

Free fatty acids are closely associated with the development of hepatic steatosis (18). Serum lipidomics revealed that a subset of species of free fatty acids were at similar levels in tempol-treated and vehicle-treated $F x r^{A I E}$ and $F x r^{A / f l}$ mice (Supplemental Figure 7A). However, serum and ileal C16:0, C18:0, C20:0, C22:0, C24:0, and C24:1 ceramide levels were significantly decreased in vehicle-treated $F x r^{I I E}$ mice compared with levels in vehicle-treated

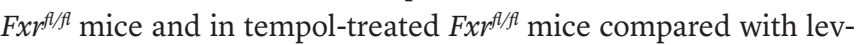
els in vehicle-treated $F x r^{A / f}$ mice (Figure 4, A and B). Mass fragmentation patterns confirmed the identity of the individual ceramide species (Supplemental Figure 8). Serum and ileal C16:0, C18:0, C20:0, C22:0, C24:0, and C24:1 ceramide levels were also els in $F x r^{I I E}$ mice were also significantly higher than in $F x r^{A / f l}$ mice (Supplemental Figure 7E). Intestinal mRNAs encoded by de novo ceramide synthesis-related genes, such as serine palmitoyltransferase, long-chain base subunit 3 (Sptlc3), ceramide synthase 4 (Cers4), degenerative spermatocyte homolog 1 (Degs1), and sphingomyelin phosphodiesterase 3 (Smpd3), were significantly lower in antibiotic-treated mice and Fxr ${ }^{A I E}$ mice on a HFD for 7 weeks and 14 weeks, respectively (Figure 5, A and B). Ceramide synthase 2 (Cers2) mRNA levels were also decreased in antibiotic-treated mice and showed a trend toward reduction $(P=0.06)$ in Fxp ${ }^{I E}$ mice, while the mRNAs encoded by genes involved in ceramide catabolism, sphingomyelin synthase 1 and 2 (Sgms1 and Sgms2) and alkaline ceramidase 1 and 3 (Acer1 and Acer3), remained similar in antibiotic-treated mice and Fxr ${ }^{\text {IIE }}$ mice compared with mRNA levels detected in controls (Figure 5, A and B).

Ceramide regulate the SREBP1C/CIDEA pathway in the liver. To establish a causal relationship between the decrease in ceramide levels and improvement of NAFLD, mice on a HFD were treated 
A

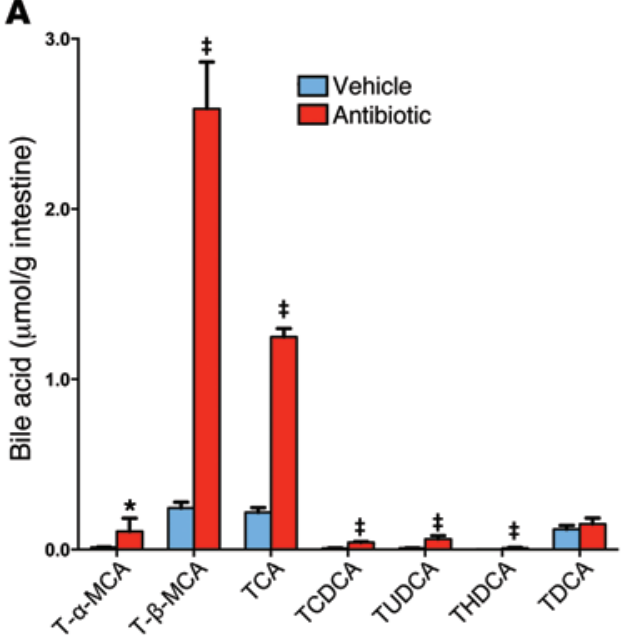

D

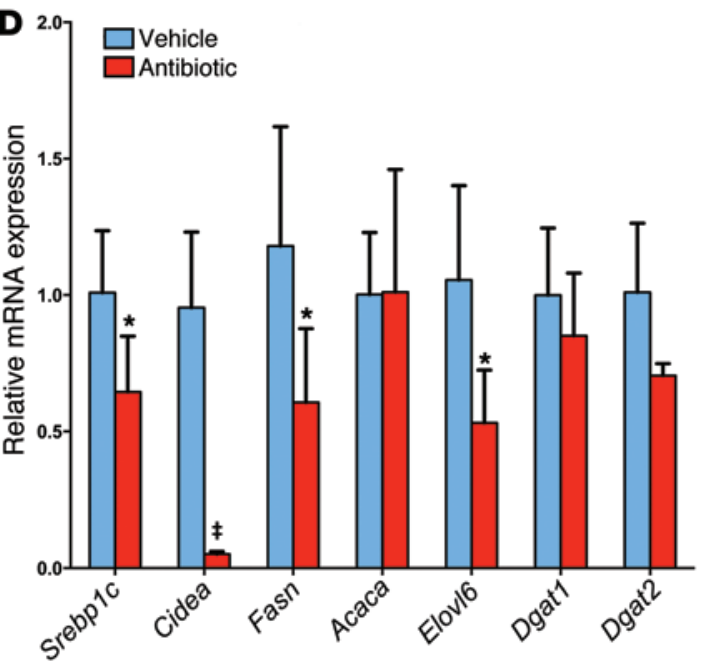

B

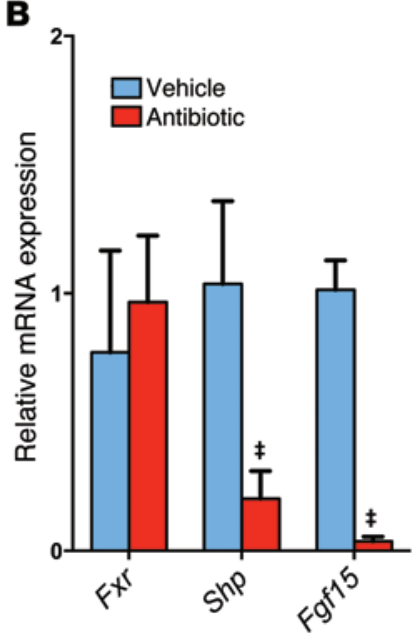

c

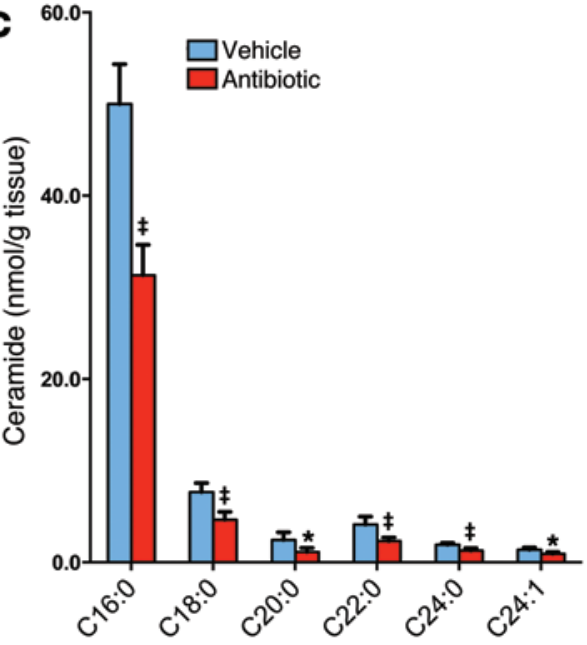

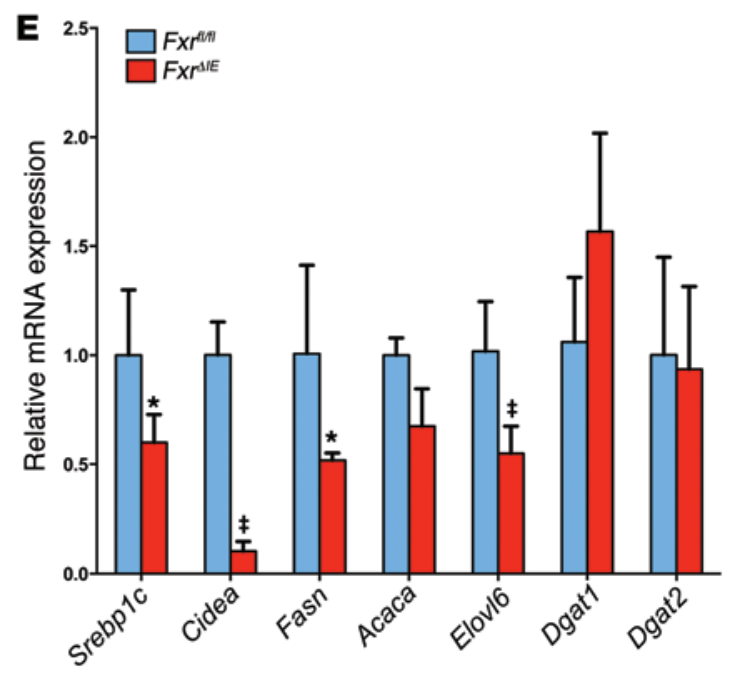

$\mathbf{F}$
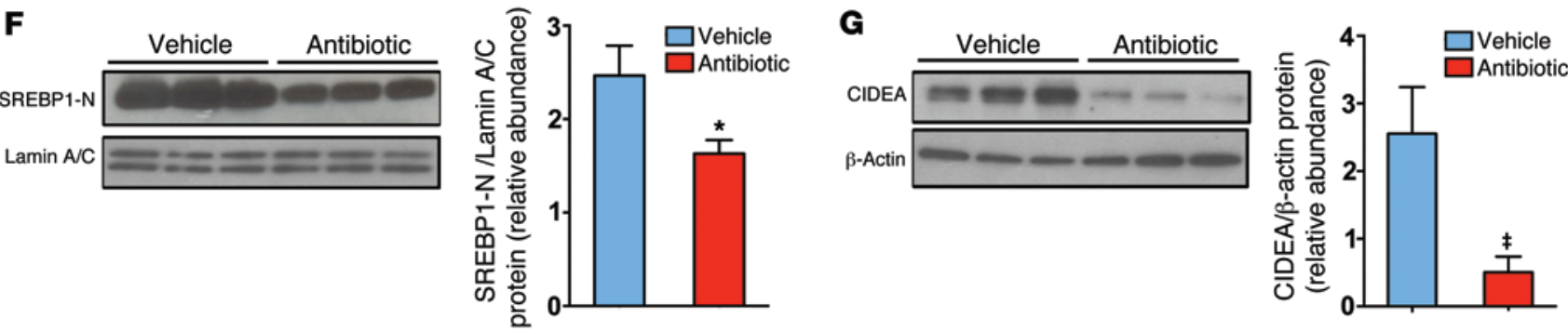

Figure 6. Intestinal FXR signaling influences the ceramide/SREBP1C/CIDEA pathway. (A) lleal bile acid profiles after 3 days of antibiotic treatment in HFD-fed mice. $n=5$ mice per group. (B) Fxr, Shp, and Fgf15 mRNA levels in the ileum after 3 days of antibiotic treatment in HFD-fed mice. $n=5$ mice per group. (C) Ileal ceramide profiles after 3 days of antibiotic treatment in HFD-fed mice. $n=5$ mice per group. (D) mRNA levels of fatty acid synthesis- and triglyceride synthesis-related enzymes in the liver of HFD-fed mice after 7 weeks of antibiotic treatment. $n=5$ mice per group. (B-D) Expression was normalized to 185 RNA. (A-D) Data are presented as the mean \pm SD. ${ }^{*} P<0.05$ and ${ }^{\ddagger} P<0.01$ (2-tailed Student's $t$ test) compared with vehicle-treated mice. (E) mRNA levels for encoding enzymes involved in fatty acid and triglyceride synthesis in the livers of $F x r^{f / f l}$ and $F x r^{4 I E}$ mice fed a HFD for 14 weeks. $n=5$ mice per group. Expression was normalized to $18 \mathrm{~S}$ RNA. Data are presented as the mean \pm SD. ${ }^{*} P<0.05$ and ${ }^{\ddagger} P<0.01$ ( 2 -tailed Student's $t$ test) compared with Fxr fl/fl mice. (F) Western blot analysis of liver nuclear SREBP1-N expression after 7 weeks of antibiotic treatment in HFD-fed mice and quantitation of SREBP1-N expression. $n=3$ mice per group. (C) Western blot analysis of liver CIDEA expression after 7 weeks of antibiotic treatment in HFD-fed mice and quantitation of SREBP1-N expression. $n=3$ mice per group. (F and $\mathbf{G}$ ) Data are presented as the mean \pm SD. ${ }^{*} P<0.05$ and ${ }^{\ddagger} P<0.01$ (2-tailed Student's $t$ test) compared with vehicle-treated mice.

with antibiotics for a short duration. Three days of antibiotic treatment did not decrease triglyceride content in the liver (Supplemental Figure 9A), and analysis of ileal bile acid composition revealed increased levels of taurine-conjugated bile acids (Figure 6A). The
FXR signaling pathway was inhibited, as revealed by lower expression of the FXR target genes $S h p$ and Fgf15, and this was correlated with decreased ceramide levels in the ileum of antibiotic-treated mice (Figure 6, B and C). These results indicated that ceramide 
A

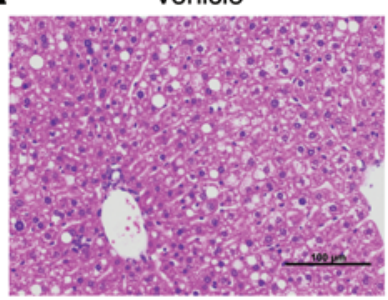

B
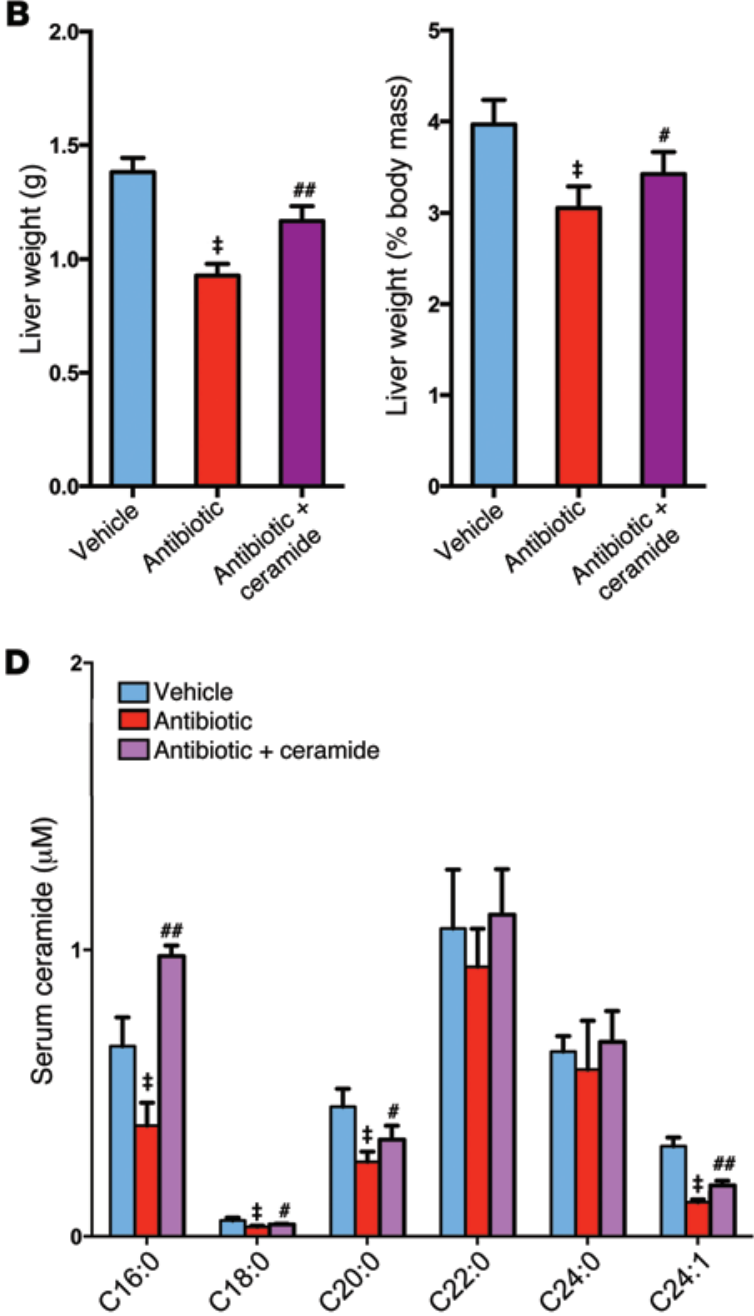

Antibiotic

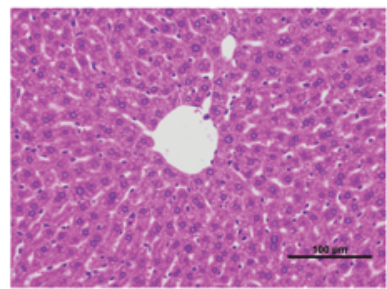

C

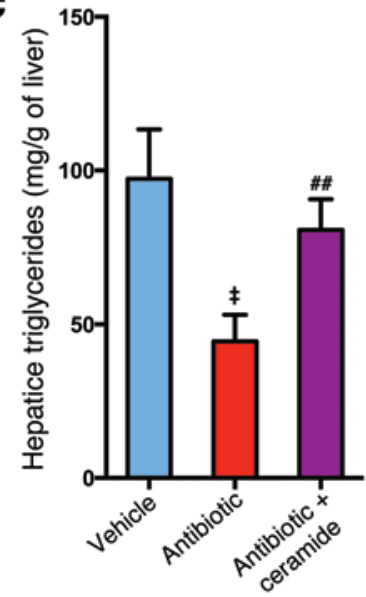

E

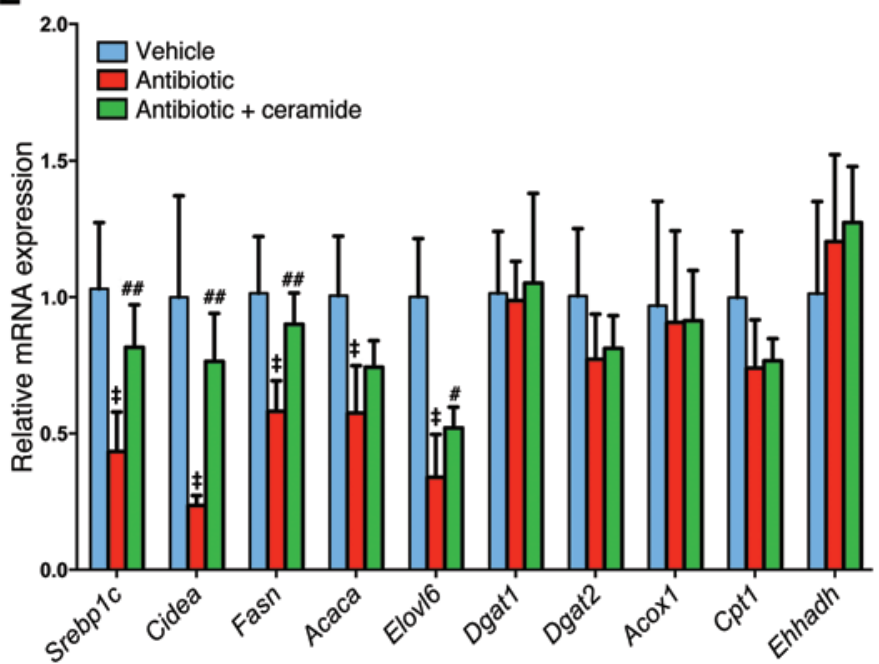

Figure 7. Administration of ceramide attenuates the effects of antibiotic treatment on the development of HFD-induced steatohepatitis. All mice were fed a HFD for 7 weeks and treated with no antibiotic (Vehicle) or a $0.1 \%$ antibiotic cocktail in their drinking water (Antibiotic). A group of mice on the antibiotic cocktail were i.p. injected with C16:0 ceramide as described in the Methods. (A) Representative H\&E staining of liver sections. $n=5$ mice per group. Scale bars: $100 \mu \mathrm{m}$. (B) Liver weights and liver weight/body weight ratios. $n=5$ mice per group. (C) Liver triglyceride content. $n=5$ mice per group. (D) Serum ceramide profiles. $n=5$ mice per group. (E) Hepatic mRNA levels of fatty acid synthesis-, triglyceride synthesis-, and fatty acid catabolism-related genes. $n=5$ mice per group. Expression was normalized to 18S RNA. All data are presented as the mean \pm SD. One-way ANOVA with Tukey's correction. ${ }^{\ddagger} P<0.01$ compared with vehicle-treated mice; ${ }^{\#} P<0.05$ and ${ }^{\# \# P}<0.01$ compared with antibiotic-treated mice.

might be causal to rather than the result of NAFLD. To test this possibility, the contribution of ceramide to NAFLD in cultured primary mouse hepatocytes was evaluated. Ceramide treatment increased triglyceride levels in primary hepatocytes in a dose-dependent manner (Supplemental Figure 9B). To elucidate the mechanisms by which ceramide leads to hepatic steatosis, the expression levels of the genes involved in hepatic lipogenesis and fatty acid oxidation were measured. Fatty acid synthesis-related genes, such as sterol response element-binding protein 1c (Srebp1c); DNA fragmentation factor $\alpha$-like effector A (Cidea); elongation of very long-chain fatty acids protein 6 (Elovl6); and triglyceride formulation-related genes, such as diacylglycerol O-acyltransferase 2 (Dgat2), were significantly upregulated by ceramide in primary hepatocytes (Supplemental Figure 9C). In contrast, the expression of genes involved 
in fatty acid $\beta$-oxidation, such as carnitine palmitoyltransferase 1 (Cpt1); acyl-coenzyme A oxidase 1 (Acox1); enoyl-coenzyme A; hydratase/3-hydroxyacyl coenzyme A dehydrogenase (Ehhadh); and acetyl-coenzyme A acyltransferase 1A (Acaa1a), were not affected by ceramide treatment (Supplemental Figure 9C). Ceramide exposure markedly induced protein levels of the mature nuclear form of SREBP1 and CIDEA (Supplemental Figure 9, D and E). In vivo, hepatic fatty acid synthesis-related genes, such as Srebp1c, Cidea, fatty acid synthase (Fasn), and Elovl6, were decreased in antibiotic-treated WT mice and in Fxr ${ }^{A I E}$ mice (Figure 6, D and E). The expression levels of genes involved in fatty acid $\beta$-oxidation remained similar in antibiotic-treated mice and Fxr ${ }^{I I E}$ mice, thus indicating that decreased fatty acid catabolism was not involved in intestinal FXR-dependent hepatic lipid accumulation (Supplemental Figure 9, F and G). Western blot analysis further revealed that the protein levels of the mature nuclear form of SREBP1 (SREBP1-N) and CIDEA were significantly downregulated in the livers of antibiotic-treated mice on a HFD for 7 weeks (Figure 6, $\mathrm{F}$ and $\mathrm{G})$. The rate-limiting enzyme cholesterol $7 \alpha$-hydroxylase (CYP7A1) initiates the classical pathway for bile acid synthesis and plays an important role in regulating lipid metabolism (20). However, Cyp7a1 mRNA levels were induced in antibiotic-treated mice, but were similar in tempol-treated mice (Supplemental Figure 9H). In addition, inflammation-related genes, such as toll-like receptor $2(T l r 2)$, toll-like receptor $4(T / r 4)$, toll-like receptor $9(\operatorname{Tl} 9)$, and TNF- $\alpha(\operatorname{Tnf} a)$, were at comparable levels in tempol- or antibiotic-treated mice (Supplemental Figure 9I). To investigate whether the decrease in ceramide is a major contributing factor in improving HFD-induced NAFLD development in antibiotic-treated mice, daily i.p. injections of purified C16:0 ceramide were administered to HFD-fed and antibiotic-treated mice. Liver histology indicated that ceramide treatment reversed the decrease in hepatic lipid droplets in antibiotic-treated mice fed a HFD for 7 weeks (Figure 7A). The liver weights, liver/body mass ratios, and hepatic triglyceride content were also significantly increased after ceramide treatment in the antibiotic-treated mice (Figure 7, B and C). Ceramide treatment also increased serum ceramide levels above those found to be reduced in the antibiotic-treated mice, and this was accompanied by an upregulation of SREBP1C/CIDEA signaling and gene expression in the livers of antibiotic-treated mice (Figure 7, $\mathrm{D}$ and E). Interestingly and unexpectedly, other ceramides were also increased in C16:0 ceramide-treated mice.

Inhibition of intestinal FXR is crucial for gut microbiome-mediated progression of NAFLD. FXr $4 I E$ mice were used to determine the role of intestinal FXR in NAFLD progression. Liver histology revealed that hepatic lipid droplets in antibiotic-treated $F x r^{f / f}$ mice, vehicle-treated Fxr ${ }^{A I E}$ mice, and antibiotic-treated $F x r^{4 I E}$ mice were significantly decreased compared with what was observed in vehicle-treated Fxr/f/l mice fed a HFD for 14 weeks (Figure 8A). Similar results were observed with tempol treatment (Supplemental Figure 10, A and B). The liver weights and liver/body mass ratios of antibiotic- and tempol-treated $F x r^{f / f l}$ mice were significantly reduced to levels similar to those seen in Fxr ${ }^{A I E}$ mice (Figure 8B and Supplemental Figure 10C). Hepatic triglyceride analysis confirmed that antibiotic or tempol treatment did not further reduce hepatic triglyceride content in Fxr ${ }^{I I E}$ mice (Figure 8C and Supplemental Figure 10D). In addition, ileal and serum C16:0, C18:0,
C20:0, C22:0, C24:0, and C24:1 ceramide levels were significantly decreased in vehicle-treated $F x r^{\triangle I E}$ mice and antibiotic-treated $F x r^{f / A}$ mice compared with those detected in vehicle-treated $F x r^{f / f l}$ mice, but were not further decreased in antibiotic-treated Fxr mice compared with vehicle-treated Fxr ${ }^{I I E}$ mice (Figure 8, D and E). In Fxr ${ }^{\Delta I E}$ mice, hepatic fatty acid synthesis-related genes, such as Srebp1c, Cidea, Fasn, and Elovl6, remained unchanged between vehicle-treated and antibiotic-treated mice (Figure 8F). Furthermore, protein levels of the mature nuclear form of SREBP1 and CIDEA were reduced in the liver after tempol treatment of Fxrt/fl mice fed a HFD for 16 weeks, whereas no further decrease was noted in Fxr ${ }^{A I E}$ mice treated with tempol (Supplemental Figure 10, $\mathrm{E}$ and $\mathrm{F}$ ). The present findings revealed that inhibition of the intestinal FXR/ceramide axis mediates the amelioration of HFDinduced NAFLD by treatment with antibiotics or tempol.

Systemic responses of mice on a HFD to tempol or antibiotic exposure. To further determine the metabolic changes associated with NAFLD, ${ }^{1} \mathrm{H}$ NMR spectroscopy was carried out on liver and cecal content extracts obtained from vehicle-, tempol-, or antibiotic-treated mice on a HFD (Supplemental Figure 11 and Supplemental Figure 12). Metabolite assignments were facilitated by published data $(21,22)$ and confirmed with a series of 2D NMR experiments (Supplemental Table 2). A total of 53 metabolites that result from the metabolism of amino acids, carbohydrates, and nucleotides were identified. 1D ${ }^{1} \mathrm{H}$ NMR spectra of the cecal contents were dominated by short-chain fatty acids (SCFAs), nucleotides, oligosaccharides, and some amino acids. Glycogen, glucose, amino acid, and nucleotide resonance signals are the dominating metabolites in the ${ }^{1} \mathrm{H}$ NMR spectra of liver.

In order to obtain the metabolic variations associated with different biological sample groups, pairwise orthogonal projections to latent structures discriminant analysis (OPLS-DA) was performed on data obtained from the cecal contents (Figure 9) or liver (Supplemental Figure 13) of tempol- or antibiotic-treated mice on a HFD for 16 weeks or 7 weeks, respectively. Differences in the metabolic profiles are displayed in the corresponding cross-validated scores plots (left) and color-coded correlation coefficient loading plots (right). The quality of these models was further validated by evaluation with coefficient of variation-ANOVA (CV-ANOVA) $(P<0.05)$ and permutation tests (200 tests) for the models (Supplemental Figures 14 and 15 and Supplemental Table 4). Here, the resonance peaks pointing upward and colored red indicate an increase in the levels of metabolites in the tempol- or antibiotic-treated mice on a HFD, whereas peaks pointing downward and colored red indicate a decrease. The statistical parameters of all the models and the main metabolic characteristics in the tempol- or antibiotic-treated groups are summarized in Supplemental Table 3. Compared with the vehicle-treated WT mice, tempol treatment decreased the levels of SCFAs (acetate, propionate, and butyrate), but elevated the levels of oligosaccharides and glucose in the cecal contents in mice on a HFD (Figure 9, A and D, and Supplemental Table 3). Similar changes were also observed in SCFAs and oligosaccharides in the cecal contents from the antibiotic-treated WT mice compared with those from the respective controls (Figure 9, C and D). However, no significant differences in the levels of SCFAs and oligosaccharides in the cecal contents from tempol-treated and vehicle-treated Fxr ${ }^{\Delta I E}$ mice were observed (Figure 9, B and D). 
A

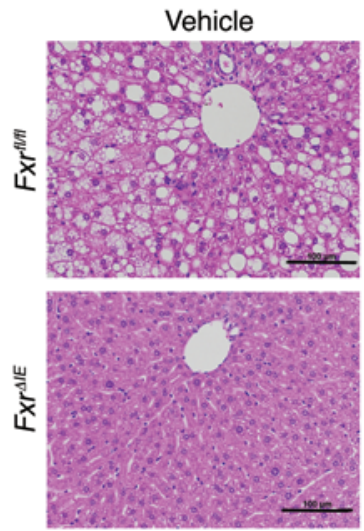

Antibiotic
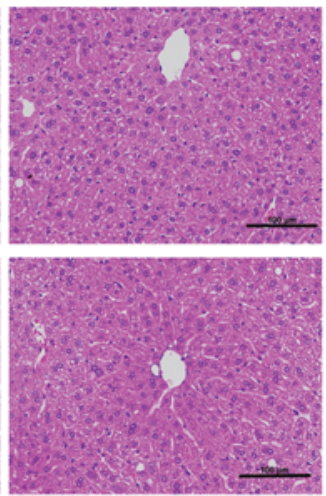
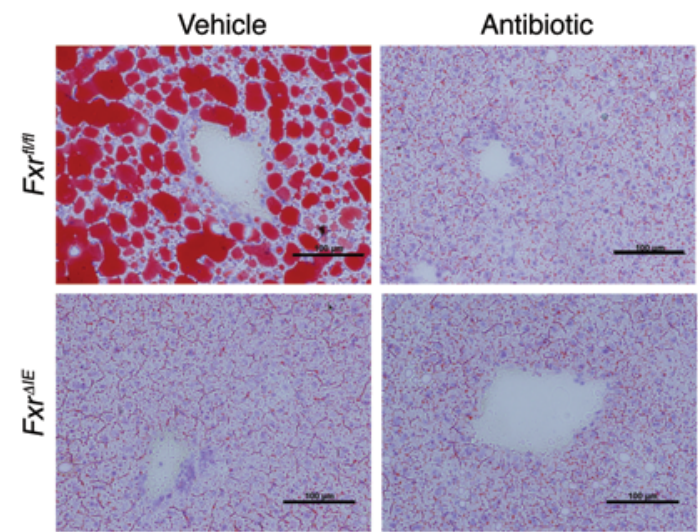
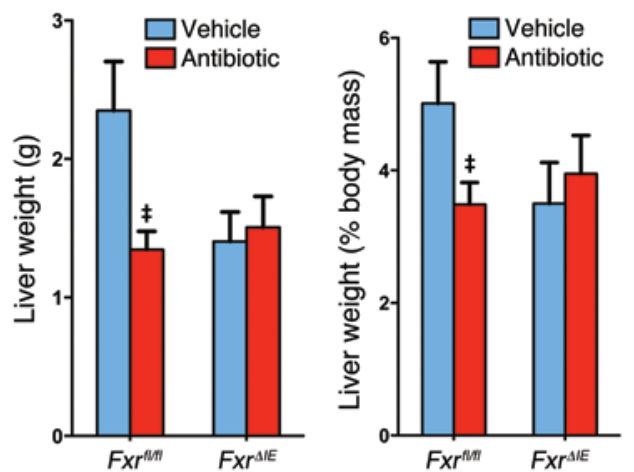

$\mathbf{C}$
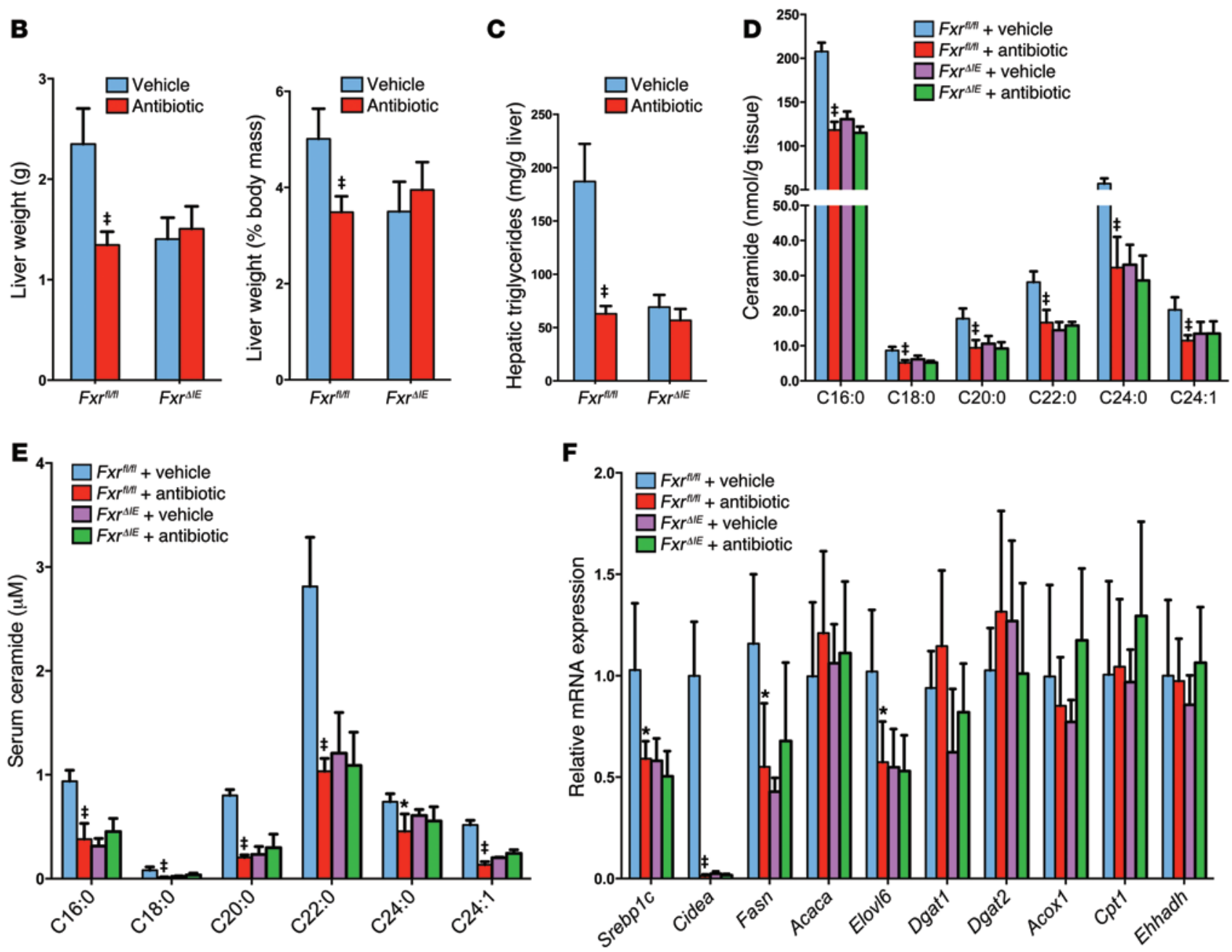

Figure 8. Antibiotic treatment improved NAFLD via the inhibition of intestinal FXR. (A) Representative H\&E staining (left panel) and Oil red 0 staining (right panel) of liver sections from vehicle- and antibiotic-treated $F x r^{f / f l}$ and $F x r^{\| I E}$ mice fed a HFD for 14 weeks. $n=5$ mice per group. Lipids stained positive (red color) with Oil red 0 . Scale bars: $100 \mu \mathrm{m}$. (B) Liver weights and liver weight/body weight ratios of vehicle- and antibiotic-treated Fxr//fl and

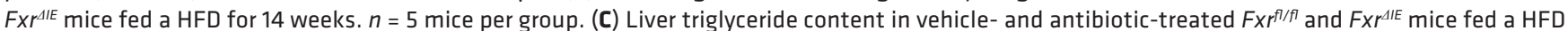
for 14 weeks. $n=5$ mice per group. (D) Ileal ceramide profiles after 14 weeks of antibiotic treatment in HFD-fed mice. $n=5$ mice per group. (E) Serum ceramide profiles after 14 weeks of antibiotic treatment in HFD-fed mice. $n=5$ mice per group. (F) mRNA levels of fatty acid synthesis-, triglyceride

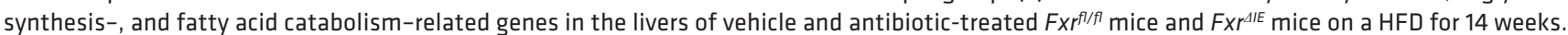
$n=5$ mice per group. (B-F) Data are presented as the mean \pm SD. One-way ANOVA with Tukey's correction. ${ }^{*} P<0.05$ and ${ }^{\ddagger} P<0.01$ compared with vehicle-treated mice of the same genotype. 
A

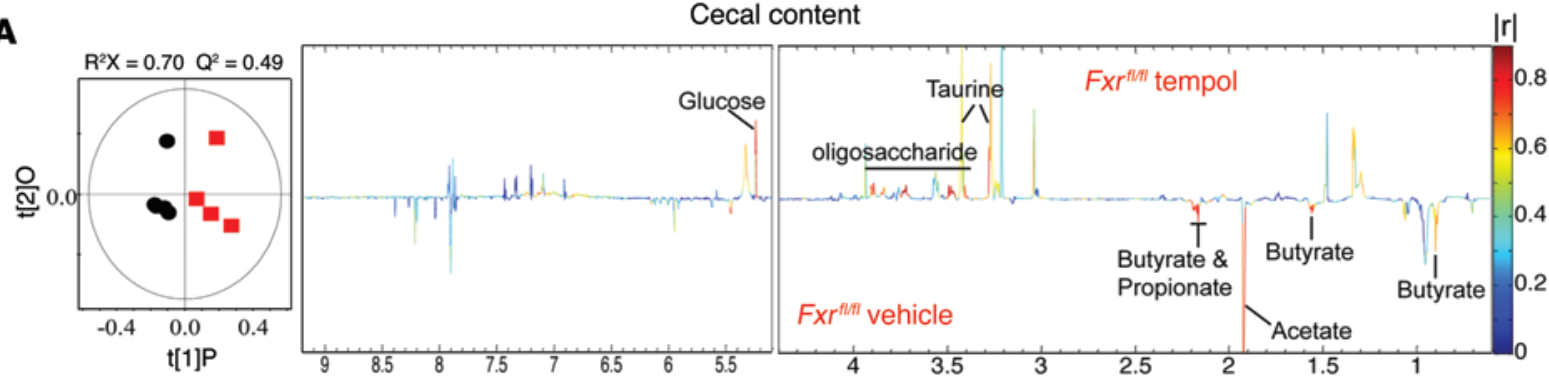

B

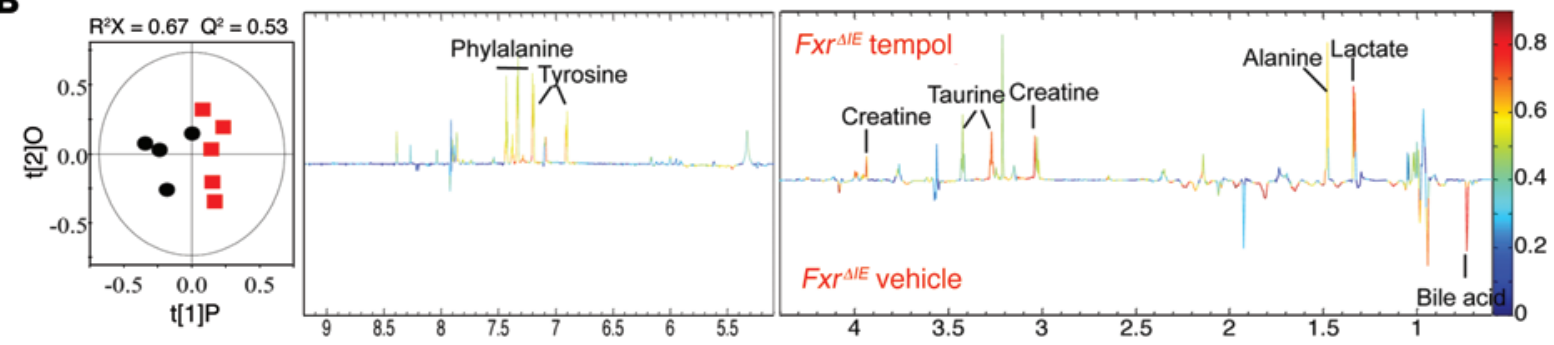

C

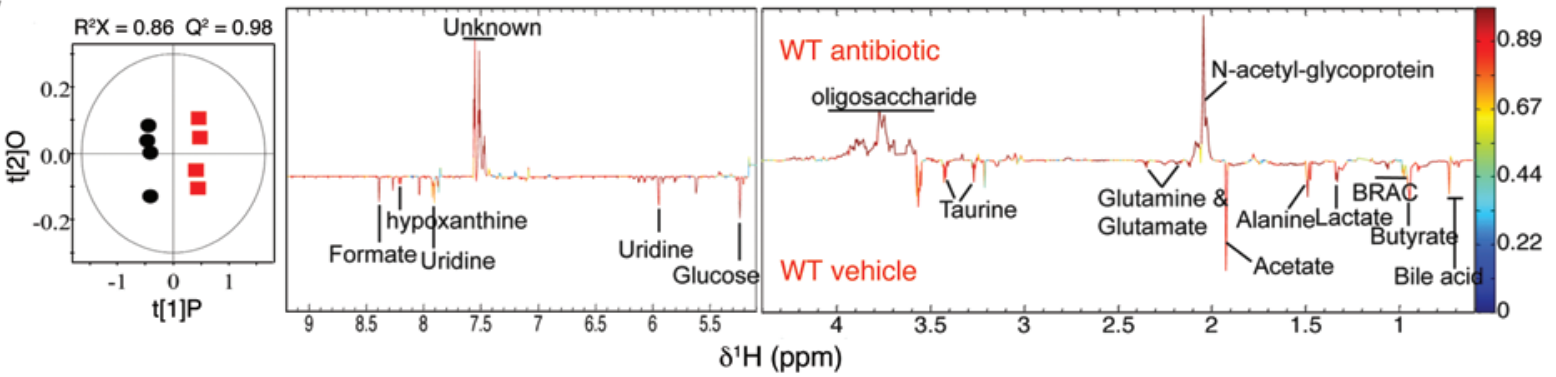

D
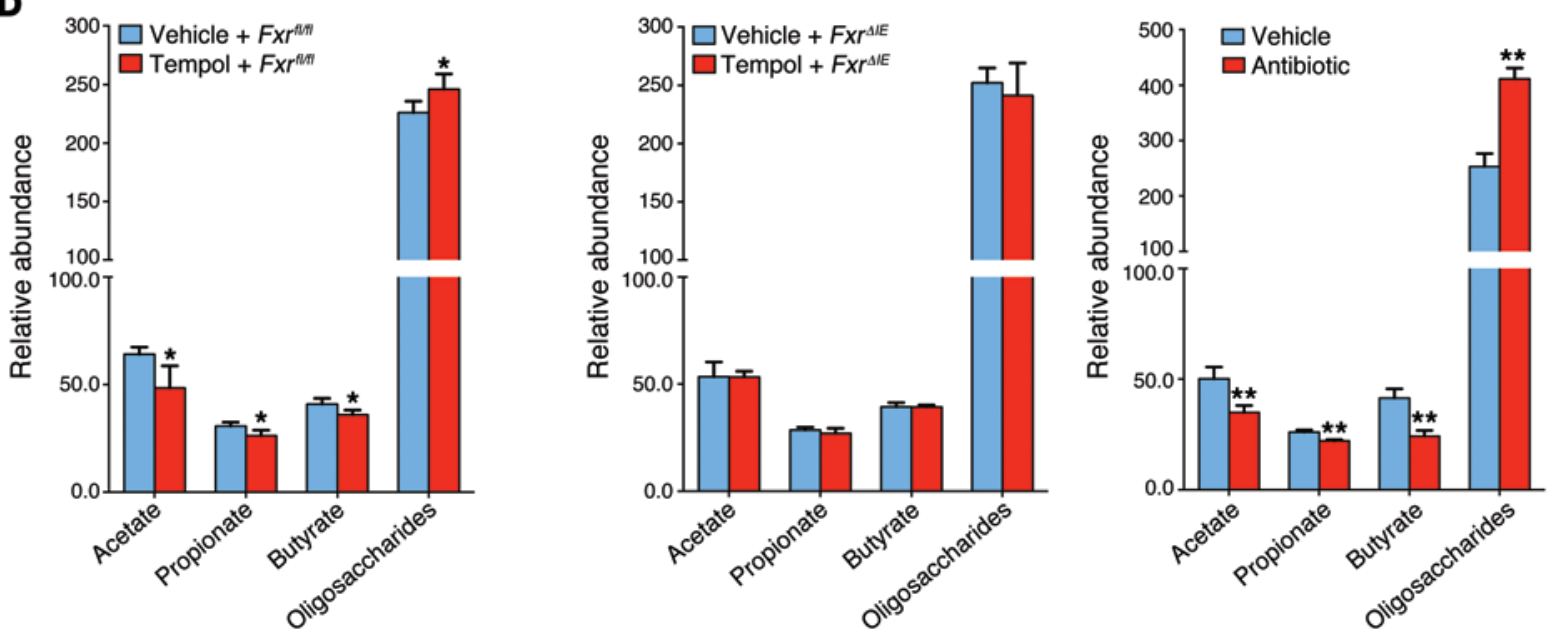

Figure 9. NMR metabolomic analysis of mouse cecal content extracts. (A) OPLS-DA scores (left) and correlation coefficient-coded loadings plots for the models (right) from NMR spectra of cecal content aqueous extracts from vehicle-treated $F x^{f / / f l}$ mice and tempol-treated $F$ r $^{f / / f l}$ mice fed a HFD for 16 weeks. $n=5$ mice per group. (B) OPLS-DA scores (left) and correlation coefficient-coded loadings plots for the models (right) from NMR spectra of cecal content aqueous extracts from vehicle-treated FXr ${ }^{\Delta I E}$ mice and tempol-treated Fxr ${ }^{\Delta I E}$ mice fed a HFD for 16 weeks. $n=4-5$ mice per group. (C) OPLS-DA scores (left) and correlation coefficient-coded loadings plots for the models (right) from NMR spectra of cecal content aqueous extracts from vehicle-treated and antibiotic-treated mice fed a HFD for 7 weeks. The correlation coefficient values indicating significantly changed metabolites are shown in Supplemental Table 3. $|r|$ cutoff value is $0.755, n=5, P<0.05$; CV-ANOVA: $P=0.04,0.02$ and $1.18 \times 10^{-4}$, respectively. $n=4-5$ mice per group. (D) Relative abundance of SCFAs (acetate, propionate, and butyrate) and oligosaccharides in cecal content extracts from vehicle-treated $F x^{f / / f l}$ mice and

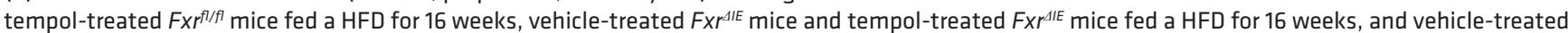
and antibiotic-treated mice fed a HFD for 7 weeks. $n=4-5$ mice per group. Data are presented as the mean \pm SD. ${ }^{*} P<0.05$, ${ }^{* *} P<0.01$ ( 2 -tailed Student's $t$ test) compared with vehicle-treated mice. 

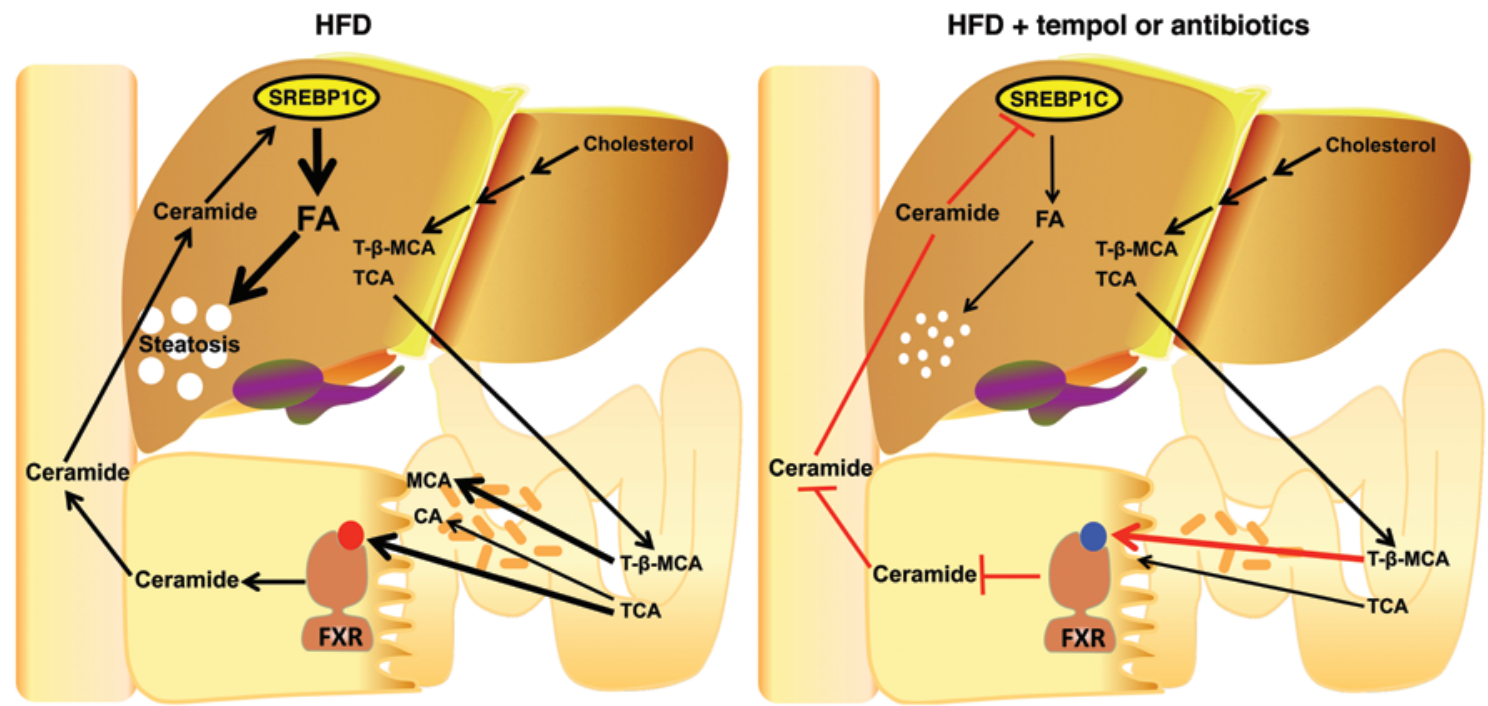

Figure 10. Summary of the gut microbiota-mediated development of NAFLD through a bile acid/intestinal FXR/ceramide axis. T- $\beta$-MCA produced in the liver from the oxidation of cholesterol was secreted into the intestine. BSH, a bacterial enzyme that hydrolyzes T- $\beta$-MCA to MCA, is substantially reduced after tempol or antibiotic treatment. T- $\beta$-MCA thereby accumulates in the ileum, which inhibits FXR signaling. The inhibition of intestinal FXR signaling elicits an improvement in mitochondrial function and repression of ceramide synthesis, resulting in decreased serum ceramide levels. Decreased circulation ceramides downregulate hepatic SREBP1C and CIDEA expression, resulting in decreased hepatic steatosis.

Tempol treatment decreased the levels of lipid and unsaturated fatty acids in the liver, whereas tempol treatment elevated the levels of glucose, glycogen, bile acids, and a range of nucleotide metabolites (e.g., uridine, hypoxanthine, and 5'-IMP), nicotinurate, and choline compared with the levels detected in vehicle-treated WT mice (Supplemental Figure 13A and Supplemental Table 3). These observations are consistent with reduced lipogenesis in the liver due to tempol treatment. However, no change in lipid or glucose metabolism in the livers of $F x r^{A I E}$ mice were observed after tempol treatment (Supplemental Figure 13B and Supplemental Table 3). In addition, antibiotic treatment markedly elevated the levels of bile acids, trimethylamine $\mathrm{N}$-oxide, choline, fumarate, formate, amino acids including branched-chain amino acids (leucine, isoleucine, and valine), alanine, glycine, tyrosine, and phenylalanine, and some nucleic acids such as hypoxanthine, uridine, and 5'-IMP in the liver (Supplemental Figure 13C and Supplemental Table 3). Compared with the vehicle-treated $F \mathrm{Fr}^{\mathrm{A} / \mathrm{fl}}$ mice, $F x r^{A I E}$ mice exhibited lower lipid and unsaturated fatty acid levels and higher taurine and glycogen levels in the liver (Supplemental Figure 13D and Supplemental Table 3).

\section{Discussion}

Antibiotic or tempol treatment of HFD-fed mice prevented NAFLD as a result of modulation of the gut microbiota, altered metabolism of bile acids, increased levels of the FXR antagonist T- $\beta$-MCA, inhibited FXR signaling in the intestine, decreased serum ceramides, and lower fatty acid synthesis in liver (Figure 10). This study is in agreement with earlier findings that germ-free mice fed a HFD were also resistant to hepatic steatosis $(6,23,24)$, thus suggesting a role for the gut microbiota in hepatic lipid accumulation. Others reported that germ-free mice colonized with intestinal bacteria from HFD-treated lines of mice that either developed or did not develop NAFLD had hepatic lipid levels similar to those of the respective donor mice, thus implicating gut bacterial composition in hepatic lipid accumulation (25). In the current study, treatment of HFD-fed germ-free mice with tempol did not decrease hepatic lipids in germ-free mice, although these lipid levels were already lower in this mouse model compared with those detected in normal WT mice $(38 \mathrm{mg} / \mathrm{g}$ vs. 70-100 mg/g liver for HFD-fed germfree and WT mice, respectively) (L. Zhang, J. Cai, C. Xie, and A.D. Patterson, unpublished observations). This lower hepatic lipid accumulation in HFD-fed germ-free mice is likely due to the high levels of intestinal T- $\beta$-MCA, an FXR antagonist, in these mice (11). In addition, tempol did not reduce further hepatic triglyceride levels beyond those achieved by antibiotic treatment of HFD-fed WT mice. A role for intestinal FXR in mediating the effects of antibiotic or tempol treatment on NAFLD was further supported by the finding that Fxp ${ }^{I I E}$ mice had much lower HFD-induced triglyceride accumulation in the liver than $\operatorname{did} F x^{f / / A}$ mice. Although they had minimal HFD-induced hepatic steatosis, tempol or antibiotics did not alter hepatic triglyceride levels in $F x r^{4 I E}$ mice. The finding that both mice lacking intestinal FXR and germ-free mice were resistant to HFD-induced hepatosteatosis further support the view that FXR signaling, the gut microbiota, and taurine-conjugated bile acids are all involved in controlling hepatic lipid accumulation. The current mechanistic studies further established that mitochondrial complex II activity was elevated in the ileum of Fxr ${ }^{I I E}$ mice. Levels of a subset of ceramides were decreased in ileum and serum from Fxr ${ }^{I I E}$ mice, and this was accompanied by downregulation of SREBP1/CIDEA signaling in the liver. The possibility cannot be ruled out that lower FXR signaling in response to modulation of the microbiota with tempol or antibiotics is due to other conjugated bile acid metabolites or other metabolic changes that result from the modified gut microbiota population.

As noted above, NAFLD is tightly associated with alterations in the composition of the microbiota. Roux-en-Y gastric bypass 
leads to marked changes in the gut microbiota population in mice after weight-loss surgery as compared with that seen in sham-operated mice, and these changes correlate with decreased obesity and fatty liver (26-29). Consistent with these studies, the present results revealed that tempol treatment reduced the phylum Firmicutes and markedly increased the phylum Proteobacteria in mice on a HFD. Associated with a reduction in the Firmicutes phylum (7), a significant reduction in bacterial fermentation was noted in the tempol or antibiotic treatment groups based on NMR metabolomics. SCFAs, which are end-products of bacterial fermentation, account for up to $10 \%$ of caloric intake and represent an important energy source. SCFAs are known to stimulate de novo synthesis of triglycerides in the liver in part via binding to the GPCRs GPR41 (also known as FFAR3) and GPR43 (also known as FFAR2) $(30,31)$. Tempol or antibiotic treatment reduced bacterial fermentation in the intestines of WT Fxr/f/l mice, but not in those of $F x r^{I I E}$ mice. The $F x r^{f / f l}$ and $F x r^{A I E}$ mice have different bile acid compositions and different gut microbiota (16), which may account for the difference in bacterial fermentation. However, the mechanism by which intestinal FXR modulates the microbiota is not clear. 16S rRNA gene sequencing further indicated that tempol treatment markedly reduced Lactobacillus spp. In agreement with the current study, Lactobacillus gasseri and Lactobacillus johnsonii were found to be positively correlated with hepatic triglyceride accumulation (32).

NAFLD is also correlated with activation of the innate immune system. Gut-derived bacterial endotoxin LPS is carried to the liver, where it can activate TLR4 and induce the expression of proinflammatory cytokine genes such as Tnfa and Il6 via activation of the transcription factor NF-KB (33). It was proposed that LPS/TLR4 signaling is associated with the pathogenesis of NAFLD (13). Further, Tlr4-null mice revealed that TLR4 is essential for the development of hepatic steatosis (34). However, in the present study, the expression of inflammation-related genes such as Tlr2, Tlr4, Tlr9, and Tnfa were similar in both the antibiotic- and tempoltreated models. This is consistent with a recent study revealing that systemic and hepatic inflammation remained unchanged between NAFLD-resistant and NAFLD-prone microbiota profiles (25). Thus, the present findings open new perspectives on the mechanism of how the gut microbiota affect NAFLD development independently of TLR signaling.

Bile acids are critical regulators of lipid metabolism and essential for lipid absorption and cholesterol homeostasis. Bile acid sequestrant treatment was found to increased serum triglyceride levels in hypercholesterolemic patients (35). Cholic acid (CA) protected mice from hepatic lipogenesis due to the inhibition of SREBP1 and its target genes (36). In human gallstone patients, chenodeoxycholic acid (CDCA) treatment lowers elevated plasma triglyceride levels and hepatic VLDL production (37). Likewise, obeticholic acid ( $6 \alpha$-ethyl-chenodeoxycholic acid), a semisynthetic derivative of CDCA, protects against liver steatosis in Zucker (fa/ fa) obese rats (38). A phase 2a proof-of-concept trial confirmed that obeticholic acid exerts a hepatoprotective effect on fibrosis (39). In the present study, the accumulation of intestinal T- $\beta$-MCA, a natural FXR antagonist (11), in antibiotic- and tempol-treated mice likely contributed to protection from NAFLD through inhibition of intestinal FXR signaling. The present study suggests a potential role for bile acids in the progression of NAFLD. However, it should be noted that the relative concentrations of individual bile acids within the bile acid pool differ between mice and humans $(40,41)$. Notably, $\beta$-MCA is the principle bile acid in mice, while in humans the primary bile acids are CA and CDCA. Thus, in humans, it is unclear whether other bile acid conjugates function to reduce intestinal FXR activity, as occurs in T- $\beta$-MCA in mice. Additional studies will be required to determine whether the current findings in mice are translatable to humans and other species.

FXR, the central sensor and regulator for the biosynthesis and enterohepatic recycling of bile acids, plays a pivotal role in the control of lipid metabolism (42-45). Whole-body FXR-deficient mice exhibited a significant accumulation of hepatic and plasma triglyceride levels as well as of VLDL, LDL, and HDL (46, 47). Other studies revealed that the hepatic FXR/SHP pathway inhibits SREBP1C and microsomal triglyceride transfer proteinmediated (MTP-mediated) hepatic lipogenesis (48-50). However, activation of FXR with the synthetic agonist GW4064 increased HFD-induced lipid accumulation in the liver, partially due to a significant reduction in BA pool size and energy expenditure (51). Guggulsterone, a natural steroid found in the guggul plant and an FXR antagonist, was found to lower hepatic cholesterol and circulating triglyceride content and increase HDL levels $(52,53)$. These reports highlight the importance of peripheral tissue FXR in the progress of dyslipidemia, and thus tissue-specific Fxr disruption might be more informative than whole-body knockouts in determining the role of agonism and antagonism of FXR in diet-induced metabolic diseases such as NAFLD. At the tissue level, FXR is predominantly expressed in the liver and intestine, but also in other tissues such as stomach, kidney, adrenal gland, tongue, and eye $(54,55)$. A reporter mouse study further revealed constitutive FXR signaling in situ only in the terminal ileum of chow-fed mice in the absence of an exogenous FXR agonist (54), which likely reflects the presence of endogenous activators or inhibitors of FXR that may be influenced by microbiota metabolism of bile acids. In the present study, both antibiotic- and tempol-treated mouse models suggest that inhibition of intestinal FXR signaling may have a role in gut microbiota-regulated development of NAFLD and further exemplify the strong connection between the gut and liverassociated diseases. In the intestine, FXR regulated the expression of the enterokine gene Fgf15 (human Fgf19), which encodes a protein that is produced in the intestine, circulates, and signals at the liver. FGF15/19 activates hepatic FGF receptor 4 (FGFR4), Janus $\mathrm{N}$-terminal kinase (JNK), and ERK signaling, resulting in the inhibition of Cyp7a1 expression (56-58). CYP7A1 transgenic mice displayed resistance to HFD-induced hepatic lipid accumulation (59), whereas serum triglyceride levels were increased in patients with CYP7A1 deficiency (60). Cyp7a1 mRNA levels were found to be marginally increased in antibiotic-treated mice. Thus, the contribution of CYP7A1 to alleviating hepatic steatosis in the antibiotic treatment model cannot be excluded, and the potential role of CYP7A1 in the amelioration of NAFLD development by antibiotics requires further experimentation.

In the current study, a relationship between intestinal FXR signal transduction pathways and ceramide metabolism was uncovered. Lipidomics analysis revealed reduced levels of a subset of ceramides in the ileum and serum of the Fxr ${ }^{\Delta I E}$ mice and antibiotic- and tempol-treated mice, whereas free fatty acid lev- 
els remained similar. A significant reduction in hepatic ceramide levels has been a consistent finding in whole-body Fxr-null mice compared with WT mice (61). It is a widely accepted view that hepatic insulin resistance results in liver steatosis (62). Ceramides were found to inhibit insulin signaling by activation of PKC $\zeta$ and protein phosphatase $2 \mathrm{~A}(63,64)$. Pertinent to this, ceramides were linked to the pathogenesis of NAFLD through insulin resistance, oxidative stress, and inflammation $(65,66)$. Numerous reports have indicated that ceramide levels were positively associated with the degree of hepatic triglyceride content accumulation (67, 68). However, the precise mechanism by which intestinal FXR regulates ceramide synthesis metabolism remains unclear and warrants further investigation.

Hepatic steatosis is mainly due to an imbalance of de novo lipogenesis and fatty acid catabolism. The present study revealed that ceramides induced hepatic triglyceride accumulation partially via activation of the SREBP1C/CIDEA signaling pathway. No change in fatty acid $\beta$-oxidation was noted in mice treated with tempol or antibiotics, indicating that the reduction of hepatic triglyceride content was not due to increased fatty acid metabolism. This corroborates a recent finding that the SREBP1C/CIDEA signaling pathway plays a critical role in promoting hepatic lipid accumulation and in the development of hepatic steatosis (63).

In conclusion, this study revealed that intestinal FXR has a role in the progression of NAFLD. Specific ceramide species, whose levels are reduced as a result of decreased intestinal FXR signaling, may serve as a biochemical conduit between the gut and liver during the pathogenesis of fatty liver. Ceramides derived from the intestine regulate hepatic de novo fatty acid synthesis, partly via the SREBP1C/CIDEA signaling pathway. Thus, selective modulation of FXR in the intestine may represent a promising therapeutic strategy to treat NAFLD.

\section{Methods}

Materials and reagents. Tempol and BNS were purchased from SigmaAldrich. Bile acids were obtained from Steraloids Inc. and SigmaAldrich, and taurocholic acid-d5 sodium salt was purchased from Toronto Research Chemicals Inc. Ceramides were obtained from Avanti Polar Lipids. HFDs (60 kcal\% fat) were purchased from BioServ. T- $\beta$-MCA was synthesized according to the scheme shown in Supplemental Methods.

Animal studies. Intestine-specific Fxr-null $\left(\right.$ Fxr $\left.{ }^{4 I E}\right)$ mice and WT $\left(F x r^{f / f l}\right)$ mice were on a C57BL/6N genetic background. Fxr $r^{f / f l}$ and $F x r^{A I E}$ (43) mice were generated by backcrossing with C57BL/6N mice for over 10 generations. Fx $r^{f / f l}$ and Fxr $r^{A I E}$ mice were littermates of more than 10 generations and were cohoused, thus the microbiomes were identical. When the $\mathrm{Fxr}^{\mathrm{Al} / \mathrm{f}}$ and $\mathrm{Fxr}^{\mathrm{AIE}}$ mice were fed a HFD and treated with tempol or antibiotics, they were then separated into different cages. For the antibiotic (the combination of BNS) study, male C57BL/6N mice, from 6 weeks of age, were fed a HFD and administered $0.1 \%(w / v)$ of each compound (the combination of BNS) in their drinking water. For the tempol study, male C57BL/6N mice, from 6 weeks of age, were fed a HFD and administered $0.064 \%(\mathrm{w} / \mathrm{v})$ tempol in their drinking water. For the tempol-treated, antibiotic-depleted mouse study, 6-week-old male C57BL/6N mice fed a HFD were divided into 4 groups: vehicle; tempol (0.064\% tempol in the drinking water); antibiotic ( $0.1 \%$ antibiotic cocktail in the drinking water); and tempol plus antibiotic $(0.064 \%$ tempol plus $0.1 \%$ antibiotic cocktail in the drinking water) and treated for 16 weeks. For the ceramide-treated antibiotic-depleted mouse study, 6-week-old male C57BL/6N mice fed a HFD were divided into 3 groups: vehicle; antibiotic ( $0.1 \%$ antibiotic cocktail in the drinking water); and ceramide plus antibiotic ( $0.1 \%$ antibiotic cocktail in the drinking water) and treated for 7 weeks. C16:0 ceramide (Avanti Polar Lipids) was dissolved in $0.9 \%$ saline with $0.5 \% \mathrm{CMCNa}$ and $5 \%$ Tween 80 . The mice were administered C16:0 ceramide at a dose of $10 \mathrm{mg} / \mathrm{kg} /$ day by i.p. injection; $0.9 \%$ saline with $0.5 \% \mathrm{CMCNa}$ and $5 \%$ Tween 80 was injected into control animals. For the in vivo T- $\beta$-MCA studies, 6-week-old male C57BL/6N mice were fed a HFD and treated with antibiotics ( $0.1 \%$ of each compound of the BNS combination) for 3 days. Vehicle (saline), TCA (400 mg/kg body weight, dissolved in saline), or a combination of TCA and T- $\beta$-MCA ( $400 \mathrm{mg} / \mathrm{kg}$ body weight of each compound, dissolved in saline) was orally administered to mice followed by a second dose 22 hours later, and the mice were killed 2 hours after the last dose for tissue collection.

Preparation and culture of primary hepatocytes, RNA analysis, and Western blot analysis. See Supplemental Methods for a detailed description.

$16 S$ rRNA gene sequencing of the intestinal microbiome and data analysis. See Supplemental Methods for a detailed description. The $16 \mathrm{~S}$ rRNA sequencing data are deposited in the NCBI's Gene Expression Omnibus (GEO PRJNA268203).

Metabolomics analysis. The sample preparation for lipidomics analysis is described in the Supplemental Methods. For lipidomics discovery, samples were analyzed by UPLC-ESI-QTOFMS using a Waters Acquity CSH $1.7-\mu \mathrm{m} \mathrm{C18}$ column $(2.1 \times 100 \mathrm{~mm})$ under the following conditions: UPLC: A, acetonitrile/water (60:40); B, isopropanol/acetonitrile (90:10); both A and B contained $10 \mathrm{mM}$ ammonium acetate and $0.1 \%$ formic acid. Gradient: initial $60 \%$ A to $57 \%$ A at 2 minutes, to $50 \% \mathrm{~A}$ at 2.1 minutes ${ }^{*}$, to $46 \% \mathrm{~A}$ at 12 minutes, to $30 \% \mathrm{~A}$ at 12.1 minutes ${ }^{*}$, to $1 \% \mathrm{~A}$ at 18 minutes before returning to initial conditions at 18.5 minutes with equilibration for 2 additional minutes (asterisk indicates the ballistic gradient). The flow rate was $0.4 \mathrm{ml} /$ minute. The column temperature was maintained at $55^{\circ} \mathrm{C}$. For MS, the same conditions were applied as above, except the run time was 18 minutes.

Quantification of lipid markers (ceramides) was performed by multiple reaction monitoring (MRM) and/or parent ion scanning using a Waters UPLC Acquity system coupled to a Waters Xevo TQ MS. A Waters Acquity BEH C18 column $(2.1 \times 100 \mathrm{~mm})$ was used. UPLC was performed with the following: $\mathrm{A}$, water; $\mathrm{B}$, acetonitrile/isopropylalcohol (5:2); both A and B contained $10 \mathrm{mM}$ ammonium acetate and $0.1 \%$ formic acid. Gradient: initial gradient of $70 \%$ A for 1 minute, then a linear gradient to $50 \% \mathrm{~A}$ at 3 minutes, to $1 \% \mathrm{~A}$ at 8 minutes, held until 15 minutes, then returning to initial conditions over 1 minute and held for an additional 2 minutes for column equilibration. The flow rate was $0.4 \mathrm{ml} /$ minute, and the column temperature was maintained at $50^{\circ} \mathrm{C}$. A Waters Xevo TQ was operated in MRM mode. The following instrument conditions were used: $2.2 \mathrm{kV}$ capillary voltage, $150^{\circ} \mathrm{C}$ source temperature, and a desolvation gas flow rate of $850 \mathrm{l} /$ hour at $450^{\circ} \mathrm{C}$. The total run time was 18 minutes. The cone voltage and collision energy for the MRM transitions were determined for each transition using IntelliStart software (Waters).

Quantification of bile acids was conducted on a Waters Acquity H-Class UPLC system using a Waters Acquity BEH C18 column (2.1 $\times 100 \mathrm{~mm}$ ) coupled to a Waters Xevo G2 QTOFMS. UPLC was performed with the following: $\mathrm{A}, 0.1 \%$ formic acid in water and $\mathrm{B}, 0.1 \%$ 
formic acid in acetonitrile. Gradient: initial gradient of $80 \%$ A for 4 minutes, to $60 \% \mathrm{~A}$ at 15 minutes, to $40 \% \mathrm{~A}$ at 20 minutes, to $10 \% \mathrm{~A}$ at 21 minutes, followed by flushing for 1 minute, then equilibration at initial conditions for 4 minutes. The flow rate was $0.4 \mathrm{ml} / \mathrm{minute}$, and the column temperature was maintained at $45^{\circ} \mathrm{C}$. A Waters Xevo G2 QTOF was operated in negative mode, scanning 50-850 amu at a rate of 0.3 scans/second. The following instrument conditions were used: $1.5 \mathrm{kV}$ capillary voltage, $150^{\circ} \mathrm{C}$ source temperature, $30 \mathrm{~V}$ sampling cove, and a desolvation gas flow rate of $850 \mathrm{l} /$ hour at $500^{\circ} \mathrm{C}$.

Data processing and multivariate data analysis. Chromatographic and spectral data were deconvoluted using MarkerLynx software (Waters). A multivariate data matrix containing information on sample identity, ion identity (retention time and $\mathrm{m} / \mathrm{z}$ ), and ion abundance was generated through centroiding, deisotoping, filtering, peak recognition, and integration. The intensity of each ion was calculated by normalizing the single ion counts versus total ion counts in the whole chromatogram. The data matrix was further exported into SIMCA-P software (Umetrics) and transformed by Pareto scaling, a technique that increases the importance of low-abundance ions without significant amplification of noise. Statistical models including PCA, partial least-squares discriminant analysis (PLS-DA), and OPLS-DA were established to represent the major latent variables in the data matrix.

${ }^{1} \mathrm{H}$ NMR-based metabolomics experiments. Methanol, $\mathrm{K}_{2} \mathrm{HPO}_{4}$, $\mathrm{NaH}_{2} \mathrm{PO}_{4}$ (all in analytical grade), sodium 3-trimethylsilyl [2,2,3,3-d4] propionate (TSP-d4), and D2O (99.9\% in D) were purchased from Sigma-Aldrich. Phosphate buffer $\left(0.1 \mathrm{M} \mathrm{K}_{2} \mathrm{HPO}_{4}\right.$ and $\mathrm{NaH}_{2} \mathrm{PO}_{4}, \mathrm{pH}$ 7.4) was prepared with $\mathrm{K}_{2} \mathrm{HPO}_{4}$ and $\mathrm{NaH}_{2} \mathrm{PO}_{4}$ for their good solubility and low-temperature stability. Liver tissue $(50 \mathrm{mg}$ ) was extracted 3 times with a precooled mixture of $0.6 \mathrm{ml}$ methanol and $600 \mu \mathrm{l}$ water $(2: 1$, $\mathrm{v} / \mathrm{v}$ ) using the PreCellys Tissue Homogenizer (Bertin Technologies). After centrifugation at $11,180 \mathrm{~g}$ for 10 minutes at $4^{\circ} \mathrm{C}$, the combined supernatants were dried. Each of the aqueous extracts was separately reconstituted into $600 \mu \mathrm{l}$ phosphate buffer containing 50\% D2O and $0.005 \%$ TSP-d 4 (chemical shift reference). Following centrifugation, $550 \mu \mathrm{l}$ of each extract was transferred into a 5-mm NMR tube. Cecal content samples were directly extracted using an optimized procedure described previously (22). Briefly, samples ( $50 \mathrm{mg})$ were mixed with 6001 precooled phosphate buffer, vortexed for 30 seconds, and subjected to 3 consecutive freeze-thaws followed by homogenization using the Precellys Tissue Homogenizer. After centrifugation (11,180 $g$ at $4^{\circ} \mathrm{C}$ ) for 10 minutes, the supernatants $(550 \mathrm{l})$ were transferred into 5-mm NMR tubes for NMR analysis.

${ }^{1} \mathrm{H}$ NMR spectroscopy. ${ }^{1} \mathrm{H}$ NMR spectra of aqueous liver and fecal extracts were recorded at $298 \mathrm{~K}$ on a Bruker Avance III $850 \mathrm{MHz}$ spectrometer (operating at $850.23 \mathrm{MHz}$ for ${ }^{1} \mathrm{H}$ ) equipped with a Bruker inverse cryogenic probe (Bruker Biospin). A typical 1-dimensional NMR spectrum was acquired for each of the samples using the first increment of the Nuclear Overhauser effect spectroscopy (NOESY) pulse sequence (NOESYPR1D). To suppress the water signal, weak continuous-wave irradiation was applied to the water peak during the recycle delay (2-second) and mixing time $(100 \mathrm{~ms})$. The $90^{\circ}$ pulse length was adjusted to approximately $10 \mu$ s for each sample, and 64 transients were collected into 32,000 data points for each spectrum, with a spectral width of $20 \mathrm{ppm}$. To facilitate NMR signal assignments, a range of 2D NMR spectra were acquired and processed as described previously $(33,50)$ for selected samples including ${ }^{1} \mathrm{H}-{ }^{1} \mathrm{H}$ correlation spectroscopy (COSY), ${ }^{1} \mathrm{H}-{ }^{1} \mathrm{H}$ total correlation spectroscopy (TOCSY),
${ }^{1} \mathrm{H}-{ }^{13} \mathrm{C}$ heteronuclear single-quantum correlation (HSQC), and ${ }^{1} \mathrm{H}-{ }^{13} \mathrm{C}$ heteronuclear multiple-bond correlation spectra (HMBC).

Spectral data processing and multivariate data analysis. All free induction decays (FIDs) were multiplied by an exponential function with a $1 \mathrm{~Hz}$ line-broadening factor prior to Fourier transformation. ${ }^{1} \mathrm{H}$ NMR spectra were corrected manually for phase and baseline distortions, and the spectral region $\delta 0.5-9.5$ was integrated into regions with an equal width of $0.004 \mathrm{ppm}(2.4 \mathrm{~Hz})$ using the AMIX software package (version 3.8; Bruker Biospin). The region $\delta 4.45-5.20$ was discarded by imperfect water saturation. Regions $\delta 1.15-1.23$ and $\delta 3.62-3.69$ were also removed for ethanol contamination in the cecal contents during the mouse dissection process. Each bucketed region was then normalized to the total sum of the spectral integrals to compensate for the overall concentration differences prior to statistical data analysis.

Multivariate data analysis was carried out with SIMCA-P+ software (version 13.0; Umetrics). PCA was initially performed on the NMR data to generate an overview and to assess data quality. OPLS-DA was subsequently conducted on the NMR data. The OPLS-DA models were validated using a 7-fold cross-validation method, and the quality of the model was described by the parameters R2X and Q2 values (Figures 3 and 4, and Supplemental Table 2). To facilitate interpretation of the results, back-transformation (45) of the loadings generated from the OPLS-DA was performed prior to generating the loadings plots, which were color-coded with Pearson's linear correlation coefficients of variables (or metabolites) using an in-house-developed script for MATLAB (The Mathworks Inc.). The color-coded correlation coefficient indicates the significance of the metabolite contribution to the class separation, with a "hot" color (e.g., red) being more significant than a "cold" color (e.g., blue). In this study, a cutoff value of $|r|$ greater than 0.811 ( $r>0.755$ and $r<-0.755)$ was chosen as a significant correlation coefficient based on the discrimination significance of $P$ less than or equal to 0.05 .

Bile salt hydrolase activity. See the Supplemental Methods for a detailed description.

Mitochondrial isolation and functional studies. For intestinal mitochondria, ileal mucosa was gently scraped, washed twice with PBS, minced in ice-cold mitochondrial homogenization buffer (225 mM mannitol, $75 \mathrm{mM}$ sucrose, $5 \mathrm{mM}$ MOPS, $0.5 \mathrm{mM}$ EGTA, and $2 \mathrm{mM}$ taurine [pH 7.25]) containing 0.2\% BSA, and homogenized in a loose-fitting homogenizer. For further procedure details, see the Supplemental Methods.

Histological analysis. H\&E staining was performed on formalin-fixed, paraffin-embedded sections using a standard protocol. Oil red O staining was performed on frozen liver sections using a standard protocol. At least 3 discontinuous liver sections were evaluated for each mouse.

Triglyceride content quantification. Hepatic lipids were extracted using a 2:1 chloroform/methanol solution. Liver triglycerides were measured with a triglyceride colorimetric assay kit, according to the manufacturer's recommendation (Bioassay Systems).

ATP detection. For the extraction of tissue ATP, $10 \mathrm{mg}$ ileal mucosa was homogenized with $1.0 \mathrm{ml}$ ice-cold TE-saturated phenol (SigmaAldrich). Chloroform (200 $\mu \mathrm{l})$ and deionized water $(150 \mu \mathrm{l})$ were added. The homogenate was thoroughly shaken for 20 seconds and centrifuged at $10,000 \mathrm{~g}$ for 5 minutes at $4^{\circ} \mathrm{C}$. The aliquot from the supernatant was diluted 100 -fold with deionized water, and $10 \mu \mathrm{l}$ of the diluted extract was measured using an ATP quantitation kit (Invitrogen).

Lipid absorption analysis. Mice were transferred to a metabolic cage (Jencons Scientific USA), housed individually, and adapted to the metabolic cages for 1 day. The cumulative food intake was then 
measured for 8 days. The feces were collected every 2 days for 8 days. Ileal and fecal lipids were extracted as described in the Supplemental Methods. Triglycerides were measured with a triglyceride colorimetric assay kit (Bioassay Systems). Free fatty acids (FFAs) were measured using reagents from Wako.

Statistics. Experimental values are presented as the mean \pm SD. Statistical analyses were performed using the 2-tailed Student's $t$ test and 1-way ANOVA with Tukey's confirmation. Weighted UniFrac analysis to assess changes in bacterial abundance was performed on the Galaxy web-based platform. Statistical models including PCA, PLS-DA, and OPLS-DA were established to represent the major latent variables in the data matrix. $P$ values of less than 0.05 were considered significant.

Study approval. All animal studies were performed in accordance with the Institute of Laboratory Animal Resources guidelines and approved by the IACUC of the NCI.

\section{Acknowledgments}

We thank Xin Pan and Jie Liu for help with analysis of mitochondrial isolation and functional studies, Toren Finkel for use of the
Seahorse XFe 96 analyzer, and Linda G. Byrd and John Buckley for technical assistance with the mouse studies. This work was supported by the Intramural Research Program of the Center for Cancer Research, NCI, NIH and by the National Institutes of Environmental Health Sciences (ESO22186, to A.D. Patterson). Sequencing was provided by the Penn State Genomics Core Facility (University Park, Pennsylvania, USA). This project is funded in part by a grant with the Pennsylvania Department of Health using Tobacco CURE Funds. The Department specifically disclaims responsibility for any analyses, interpretations, or conclusions of this work.

Address correspondence to: Frank J. Gonzalez, NIH, Building 37, Room 3106, Bethesda, Maryland 20892, USA. Phone: 301.496.9067; E-mail: gonzalef@mail.nih.gov. Or to: Andrew D. Patterson, Center for Molecular Toxicology and Carcinogenesis, Department of Veterinary and Biomedical Sciences, 322 Life Sciences Building, University Park, Pennsylvania 16802, USA. Phone: 814.867.4565; E-mail: adp117@psu.edu.
1. Sattar N, Forrest E, Preiss D. Non-alcoholic fatty liver disease. BMJ. 2014;349:g4596.

2. Dyson J, Day C. Treatment of non-alcoholic fatty liver disease. Dig Dis. 2014;32(5):597-604.

3. Bhatia LS, Curzen NP, Calder PC, Byrne CD. Non-alcoholic fatty liver disease: a new and important cardiovascular risk factor? Eur Heart J. 2012;33(10):1190-1200.

4. Baffy G, Brunt EM, Caldwell SH. Hepatocellular carcinoma in non-alcoholic fatty liver disease: an emerging menace. J Hepatol. 2012; 56(6):1384-1391.

5. Park JS, Seo JH, Youn HS. Gut microbiota and clinical disease: obesity and nonalcoholic Fatty liver disease. Pediatr Gastroenterol Hepatol Nutr. 2013;16(1):22-27.

6 . Backhed F, et al. The gut microbiota as an environmental factor that regulates fat storage. Proc Natl Acad Sci U S A . 2004;101(44):15718-15723.

7. Turnbaugh PJ, Ley RE, Mahowald MA, Magrini V, Mardis ER, Gordon JI. An obesity-associated gut microbiome with increased capacity for energy harvest. Nature. 2006;444(7122):1027-1031.

8. Dumas ME, et al. Metabolic profiling reveals a contribution of gut microbiota to fatty liver phenotype in insulin-resistant mice. Proc Natl Acad Sci U S A. 2006;103(33):12511-12516.

9. Spencer MD, Hamp TJ, Reid RW, Fischer LM, Zeisel SH, Fodor AA. Association between composition of the human gastrointestinal microbiome and development of fatty liver with choline deficiency. Gastroenterology. 2011;140(3):976-986.

10. Compare D, et al. Gut - liver axis: the impact of gut microbiota on non alcoholic fatty liver disease. Nutr Metab Cardiovasc Dis. 2012;22(6):471-476.

11. Sayin SI, et al. Gut microbiota regulates bile acid metabolism by reducing the levels of tauro- $\beta$ muricholic acid, a naturally occurring FXR antagonist. Cell Metab. 2013;17(2):225-235.

12. Li F, et al. Microbiome remodelling leads to inhibition of intestinal farnesoid $\mathrm{X}$ receptor signalling and decreased obesity. Nat Commun. 2013;4:2384.
13. Aron-Wisnewsky J, Gaborit B, Dutour A, Clement K. Gut microbiota and non-alcoholic fatty liver disease: new insights. Clin Microbiol Infec. 2013;19(4):338-348.

14. Fuchs C, Claudel T, Trauner M. Bile acid-mediated control of liver triglycerides. Semin Liver Dis. 2013;33(4):330-342.

15. Karlsson CL, Onnerfalt J, Xu J, Molin G, Ahrne $\mathrm{S}$, Thorngren-Jerneck $\mathrm{K}$. The microbiota of the gut in preschool children with normal and excessive body weight. Obesity (Silver Spring). 2012;20(11):2257-2261.

16. Ryan KK, et al. FXR is a molecular target for the effects of vertical sleeve gastrectomy. Nature. 2014;509(7499):183-188.

17. Ridlon JM, Kang DJ, Hylemon PB. Bile salt biotransformations by human intestinal bacteria. J Lipid Res. 2006;47(2):241-259.

18. Donnelly KL, Smith CI, Schwarzenberg SJ, Jessurun J, Boldt MD, Parks EJ. Sources of fatty acids stored in liver and secreted via lipoproteins in patients with nonalcoholic fatty liver disease. J Clin Invest. 2005;115(5):1343-1351.

19. Siskind LJ. Mitochondrial ceramide and the induction of apoptosis. J Bioenerg Biomembr. 2005;37(3):143-153.

20. Chiang JY. Bile acids: regulation of synthesis. JLipid Res. 2009;50(10):1955-1966.

21. Zhang L, Ye Y, An Y, Tian Y, Wang Y, Tang H. Systems responses of rats to aflatoxin $\mathrm{B} 1$ exposure revealed with metabonomic changes in multiple biological matrices. J Proteome Res. 2011;10(2):614-623.

22. Wu J, An Y, Yao J, Wang Y, Tang H. An optimised sample preparation method for NMRbased faecal metabonomic analysis. Analyst. 2010;135(5):1023-1030.

23. Backhed F, Manchester JK, Semenkovich CF, Gordon JI. Mechanisms underlying the resistance to diet-induced obesity in germ-free mice. Proc Natl Acad Sci U S A. 2007;104(3):979-984.

24. Rabot S, et al. Germ-free C57BL/6J mice are resistant to high-fat-diet-induced insulin resis- tance and have altered cholesterol metabolism. FASEB J. 2010;24(12):4948-4959.

25. Le Roy $\mathrm{T}$, et al. Intestinal microbiota determines development of non-alcoholic fatty liver disease in mice. Gut. 2013;62(12):1787-1794.

26. Zhang $\mathrm{H}$, et al. Human gut microbiota in obesity and after gastric bypass. Proc Natl Acad Sci US A. 2009;106(7):2365-2370.

27. Furet JP, et al. Differential adaptation of human gut microbiota to bariatric surgeryinduced weight loss: links with metabolic and low-grade inflammation markers. Diabetes. 2010;59(12):3049-3057.

28. Li JV, et al. Metabolic surgery profoundly influences gut microbial-host metabolic cross-talk. Gut. 2011;60(9):1214-1223.

29. Liou AP, Paziuk M, Luevano J-M, Machineni S, Turnbaugh PJ, Kaplan LM. Conserved shifts in the gut microbiota due to gastric bypass reduce host weight and adiposity. Sci Transl Med. 2013;5(178):178ra141.

30. Cox LM, Blaser MJ. Pathways in microbe-induced obesity. Cell Metab. 2013;17(6):883-894.

31. Tremaroli V, Bäckhed F. Functional interactions between the gut microbiota and host metabolism. Nature. 2012;489(7415):242-249.

32. Zeng H, Liu J, Jackson MI, Zhao F-Q, Yan L, Combs GF. Fatty liver accompanies an increase in lactobacillus species in the hind gut of C57BL/6 mice fed a high-fat diet. J Nutr. 2013;143(5):627-631.

33. Benhamed $\mathrm{F}$, et al. The lipogenic transcription factor ChREBP dissociates hepatic steatosis from insulin resistance in mice and humans. J Clin Invest. 2012;122(6):2176-2194.

34. Ye D, et al. Toll-like receptor- 4 mediates obesityinduced non-alcoholic steatohepatitis through activation of X-box binding protein-1 in mice. Gut. 2012;61(7):1058-1067.

35. Insull W Jr . Clinical utility of bile acid sequestrants in the treatment of dyslipidemia: a scientific review. South Med J. 2006;99(3):257-273.

36. Watanabe $\mathrm{M}$, et al. Bile acids lower triglyceride 
levels via a pathway involving FXR, SHP, and SREBP-1c. J Clin Invest. 2004;113(10):1408-1418.

37. Leiss O, Von Bergmann K. Different effects of chenodeoxycholic acid and ursodeoxycholic acid on serum lipoprotein concentrations in patients with radiolucent gallstones. Scand J Gastroenterol. 1982;17(5):587-592.

38. Cipriani S, Mencarelli A, Palladino G, Fiorucci S. FXR activation reverses insulin resistance and lipid abnormalities and protects against liver steatosis in Zucker (fa/fa) obese rats. J Lipid Res. 2010;51(4):771-784

39. Mudaliar S, et al. Efficacy and safety of the farnesoid $\mathrm{X}$ receptor agonist obeticholic acid in patients with type 2 diabetes and nonalcoholic fatty liver disease. Gastroenterology. 2013;145(3):574-582.e1.

40. Hofmann AF, Schteingart CD, Hagey LR. Species differences in bile acid metabolism. In: Paumgartner G, Beuers U, eds. Bile Acids In Liver Diseases. Boston, Massachusetts, USA: Kluwer Academic Publishers; 1995:3-30.

41. Krasowski MD, Yasuda K, Hagey LR, Schuetz EG. Evolution of the pregnane $\mathrm{x}$ receptor: adaptation to cross-species differences in biliary bile salts. Mol Endocrinol. 2005;19(7):1720-1739.

42. Sinal CJ, Tohkin M, Miyata M, Ward JM, Lambert G, Gonzalez FJ. Targeted disruption of the nuclear receptor FXR/BAR impairs bile acid and lipid homeostasis. Cell. 2000;102(6):731-744.

43. Kim I, et al. Differential regulation of bile acid homeostasis by the farnesoid $\mathrm{X}$ receptor in liver and intestine. JLipid Res. 2007;48(12):2664-2672.

44. Matsubara T, Li F, Gonzalez FJ. FXR signaling in the enterohepatic system. Mol Cell Endocrinol. 2013;368(1):17-29.

45. Seyer $\mathrm{P}$, et al. Hepatic glucose sensing is required to preserve beta cell glucose competence. JClin Invest. 2013;123(4):1662-1676.

46. Zhang Y, et al. Activation of the nuclear receptor FXR improves hyperglycemia and hyperlipidemia in diabetic mice. Proc Natl Acad Sci U S A. 2006;103(4):1006-1011.

47. Ma K, Saha PK, Chan L, Moore DD. Farnesoid X receptor is essential for normal glucose homeo- stasis. J Clin Invest. 2006;116(4):1102-1109.

48. Hirokane H, Nakahara M, Tachibana S, Shimizu M, Sato R. Bile acid reduces the secretion of very low density lipoprotein by repressing microsomal triglyceride transfer protein gene expression mediated by hepatocyte nuclear factor-4. J Biol Chem. 2004;279(44):45685-45692.

49. Zhu Y, Li F, Guo GL. Tissue-specific function of farnesoid $\mathrm{X}$ receptor in liver and intestine. Pharmacol Res. 2011;63(4):259-265.

50. Anakk S, Watanabe M, Ochsner SA, McKenna NJ, Finegold MJ, Moore DD. Combined deletion of Fxr and Shp in mice induces Cyp17a1 and results in juvenile onset cholestasis. JClin Invest. 2011;121(1):86-95.

51. Watanabe $\mathrm{M}$, et al. Lowering bile acid pool size with a synthetic farnesoid X receptor (FXR) agonist induces obesity and diabetes through reduced energy expenditure. J Biol Chem. 2011;286(30):26913-26920.

52. Urizar NL, et al. A natural product that lowers cholesterol as an antagonist ligand for FXR. Science. 2002;296(5573):1703-1706.

53. Cui J, et al. Guggulsterone is a farnesoid X receptor antagonist in coactivator association assays but acts to enhance transcription of bile salt export pump. Jiol Chem. 2003;278(12):10214-10220.

54. Houten SM, Volle DH, Cummins CL, Mangelsdorf DJ, Auwerx J. In vivo imaging of farnesoid $\mathrm{X}$ receptor activity reveals the ileum as the primary bile acid signaling tissue. Mol Endocrinol. 2007;21(6):1312-1323.

55. Higashiyama H, Kinoshita M, Asano S. Immunolocalization of farnesoid $\mathrm{X}$ receptor (FXR) in mouse tissues using tissue microarray. Acta Histochem. 2008;110(1):86-93.

56. Song KH, Li T, Owsley E, Strom S, Chiang JY. Bile acids activate fibroblast growth factor 19 signaling in human hepatocytes to inhibit cholesterol 7alpha-hydroxylase gene expression. Hepatology. 2009;49(1):297-305.

57. Inagaki T, et al. Fibroblast growth factor 15 functions as an enterohepatic signal to regulate bile acid homeostasis. Cell Metab. 2005;2(4):217-225 58. Kong B, Wang L, Chiang JY, Zhang Y, Klaassen
CD, Guo GL. Mechanism of tissue-specific farnesoid $\mathrm{X}$ receptor in suppressing the expression of genes in bile-acid synthesis in mice. Hepatology. 2012;56(3):1034-1043.

59. Li T, Owsley E, Matozel M, Hsu P, Novak CM, Chiang JY. Transgenic expression of cholesterol $7 \alpha$-hydroxylase in the liver prevents high-fat dietinduced obesity and insulin resistance in mice. Hepatology. 2010;52(2):678-690.

60. Pullinger CR, et al. Human cholesterol $7 \alpha$-hydroxylase (CYP7A1) deficiency has a hypercholesterolemic phenotype. J Clin Invest. 2002;110(1):109-117.

61. Matsubara T, Tanaka N, Patterson AD, Cho JY, Krausz KW, Gonzalez FJ. Lithocholic acid disrupts phospholipid and sphingolipid homeostasis leading to cholestasis in mice. Hepatology. 2011;53(4):1282-1293.

62. Farese RV Jr, Zechner R, Newgard CB, Walther TC. The problem of establishing relationships between hepatic steatosis and hepatic insulin resistance. Cell Metab. 2012;15(5):570-573.

63. Bikman BT, Summers SA. Ceramides as modulators of cellular and whole-body metabolism. JClin Invest. 2011;121(11):4222-4230.

64. Russo SB, et al. Ceramide synthase 5 mediates lipidinduced autophagy and hypertrophy in cardiomyocytes. J Clin Invest. 2012;122(11):3919-3930.

65. Pagadala M, Kasumov T, McCullough AJ, Zein NN, Kirwan JP. Role of ceramides in nonalcoholic fatty liver disease. Trends Endocrinol Metab. 2012;23(8):365-371.

66. Holland WL, et al. Lipid-induced insulin resistance mediated by the proinflammatory receptor TLR4 requires saturated fatty acid-induced ceramide biosynthesis in mice. JClin Invest. 2011;121(5):1858-1870.

67. Yetukuri L, Katajamaa M, Medina-Gomez G, Seppänen-Laakso T, Vidal-Puig A, Orešič M. Bioinformatics strategies for lipidomics analysis: characterization of obesity related hepatic steatosis. BMC Syst Biol. 2007;1:12.

68. Rotolo J, et al. Anti-ceramide antibody prevents the radiation gastrointestinal syndrome in mice. JClin Invest. 2012;122(5):1786-1790. 